\title{
Methodological Aspects of Randomized Controlled Trials for Tinnitus: A Systematic Review and How a Decision Support System Could Overcome Barriers
}

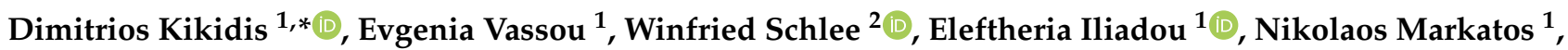 \\ Aikaterini Triantafyllou ${ }^{1}$ and Berthold Langguth ${ }^{2}$ \\ 1 First Department of Otorhinolaryngology, Head and Neck Surgery, Hippokration General Hospital, \\ National and Kapodistrian University of Athens, 15772 Athens, Greece; evassou@med.uoa.gr (E.V.); \\ iliadoue@med.uoa.gr (E.I.); markatosn84@gmail.com (N.M.); katerinatriant18@gmail.com (A.T.) \\ 2 Department of Psychiatry and Psychotherapy, Universität Regensburg, 93053 Regensburg, Germany; \\ winfried.schlee@gmail.com (W.S.); berthold.langguth@medbo.de (B.L.) \\ * Correspondence: dimitriskikidis@yahoo.com
}

\section{check for} updates

Citation: Kikidis, D.; Vassou, E.; Schlee, W.; Iliadou, E.; Markatos, N.; Triantafyllou, A.; Langguth, B.

Methodological Aspects of

Randomized Controlled Trials for

Tinnitus: A Systematic Review and How a Decision Support System Could Overcome Barriers. J. Clin. Med. 2021, 10, 1737. https://doi.org/ $10.3390 /$ jcm 10081737

Academic Editor: Christof Röösli

Received: 1 March 2021

Accepted: 8 April 2021

Published: 16 April 2021

Publisher's Note: MDPI stays neutral with regard to jurisdictional claims in published maps and institutional affiliations.

Copyright: (c) 2021 by the authors. Licensee MDPI, Basel, Switzerland. This article is an open access article distributed under the terms and conditions of the Creative Commons Attribution (CC BY) license (https:// creativecommons.org/licenses/by/ $4.0 /)$.

\begin{abstract}
Although a wide range of tinnitus management interventions is currently under research and a variety of therapeutic interventions have already been applied in clinical practice, no optimal and universal tinnitus treatment has been reached yet. This fact is to some extent a consequence of the high heterogeneity of the methodologies used in tinnitus related clinical studies. In this manuscript, we have identified, summarized, and critically appraised tinnitus-related randomized clinical trials since 2010, aiming at systematically mapping the research conducted in this area. The results of our analysis of the 73 included randomized clinical trials provide important insight on the identification of limitations of previous works, methodological pitfalls or gaps in current knowledge, a prerequisite for the adequate interpretation of current literature and execution of future studies.
\end{abstract}

Keywords: tinnitus; tinnitus treatment; randomized controlled trial

\section{Introduction}

Tinnitus is traditionally described as the perception of a sound in the absence of corresponding external stimuli [1]. In a very recent consensus article, a more precise definition of tinnitus has been proposed: Tinnitus was defined as "the conscious awareness of a tonal and/or noise sound for which there is no identifiable corresponding external acoustic source" and tinnitus disorder was defined as "tinnitus plus tinnitus-associated emotional distress and functional disability" [2]. Tinnitus is considered an enigmatic situation and universally accepted answers to fundamental questions about its pathophysiology, course, and optimal treatment are still pending $[1,3,4]$. Its prevalence is estimated more than $10 \%$ in the general population; however, it is considered bothersome only in approximately $1 \%[3,4]$. These numbers are of paramount importance, since, according to the recently released European Tinnitus Guidelines, clinical approach and decision ma-king should take into account not only tinnitus existence but also patient's reaction to tinnitus [1]. Tinnitus is considered as a symptom well tolerated by the majority of individuals; however, it might cause levels of annoyance which can be adequate to make tinnitus the determining factor for significant impairment of the perceived health status and the overall quality of life.

One of the few things considered common ground among tinnitus community is that no optimal and universal tinnitus treatment has been reached yet [1,5]. Despite the fact that a wide range of interventions including, but not limited to, drugs and medicinal products, sound amplification, sound therapy, psychological interventions, and transcranial magnetic stimulation have been applied, none of them is universally accepted as an adequate and globally effective solution for the whole spectrum of tinnitus sufferers [1]. 
Hence, there is a pattern across the tinnitus literature according to which, a varying subgroup of responders is found in most of the studies [5]. This could be attributed to statistical variance, but it could also be claimed that some therapeutic interventions could potentially be beneficial in a specific subgroup of patients with identifiable characteristics. Very few studies, however, attempt to create and identify a certain profile correlating with treatment response and the main research question is limited to whether an intervention is effective or not in a group of patients, rather than which factors influence treatment response [6,7].

Moreover, tinnitus related literature has some specific barriers, on top of the issues identified as problematic in medical literature in general, such as sample size calculation, study settings, statistical analysis, and selection bias [8]. These drawbacks include the heterogeneity of tinnitus patients, the fluctuation in tinnitus perception, the subjective nature of tinnitus and therefore the lack of objective outcome measures, the common existence of comorbidities, as well as their interaction with tinnitus perception and the different perception of tinnitus in different cultures, as well as in different times by the same individual [9-11].

Consequently, it could be stated that the reasons for the lack of an established and effective treatment are both native and intrinsic, as well as subjective. Aim of this paper is to summarize factors, objective restraints, methodological flaws, and research insufficiencies, in order to provide some explanation for the fact that no universal tinnitus treatment has been established yet.

A systematic review of the tinnitus literature including Randomized Clinical Trial (RCTs) has been conducted aiming towards the identification of common methodological flaws and insufficiencies. Finally, a brief overview of a Decision Support System (DSS) which is the core outcome of EU-funded project Unification of Treatments and Interventions for Tinnitus Patients (UNITI) is presented [12]. The DSS takes into account epidemiological data, audiological measurements, genetic background and socioeconomic data in order to choose the optimal treatment out of the most widely used treatments (sound therapy, sound amplification, Cognitive Behavioral Therapy and Structured Counselling) at an individualized level [12].

\section{Materials and Methods}

This paper is a systematic review of literature aiming at the extraction of the characteristics of the RCTs published from 2010 to 2020 in the field of tinnitus, drafted according to PRISMA guidelines [13].

The main goal of this paper is to map and evaluate the methodology of RCTs with regards to inclusion criteria, outcome measures, consideration of confounding factors like hearing loss and whether special care was taken in order to minimize the impact of tinnitus specific confounders such as fluctuations over time or unstable treatment response. This information may lead to useful conclusions about systematic bias, methodological flaws, drawbacks, and gaps in the body of RCTs targeting tinnitus treatment.

Review question was set as following: Which were the inclusion criteria: age, characteristics of tinnitus (primary complaint, laterality, chronic/acute, onset, intermittent cha-racter, etc.), procedure, outcome measures used, study methods (sample size, power analysis, randomization, etc.), and study timeline-duration of the RCTs published between 2010 and 2020. Additionally, the research aims were formulated according to the PICO template as following:

People: Adults with history of tinnitus.

Intervention: Any kind of intervention aiming towards tinnitus treatment, care and compensation.

Comparison: Non applicable.

Outcome: Methodological aspects of tinnitus RCTs (inclusion criteria, study procedures, study outcome measures, study timeline). 


\subsection{Inclusion Criteria}

Target population consists of adults with tinnitus. Trials targeting pulsatile tinnitus were excluded. Only RCTs published between 30 June 2010 and 1 July 2020, with at least 30 participants were included. The purpose of setting these inclusion criteria was to critically examine the methodological adequacy and patterns of barriers and limitations occurring in tinnitus literature in its highest level according to their type and level of evidence provided.

\subsection{Exclusion Criteria}

Protocols of RCTs, as well as RCTs mainly targeting entities other than tinnitus, even if tinnitus could occur as symptom in their context (e.g., sudden hearing loss, Meniere disease) were excluded. Any type of study other than RCT or studies written in a language other than English were excluded.

\subsection{Information Sources}

Information sources included 3 major databases (Medline, Central, and Scopus). All searches were conducted by two authors independently. The results were then handsearched [14].

\subsection{Search}

The search syntax used in Medline was broad: "tinnitus AND random*". Similar approach was used in the other two databases. Filters applied were "English language", "10 years" and "Randomized Controlled Trial".

\subsection{Study Selection}

Studies obtained from the aforementioned search have been reviewed independently by two authors. In that stage of analysis, the authors have identified duplicates or multiple reports of the same study, by first examining the titles and abstracts of the yielded studies and then their full text. Results of grey literature, trial registration platforms, and conferences were then added. The senior author resolved any disagreements.

\subsection{Data Collection Process}

Two reviewers have screened full-text articles and produced a matrix of relevant data independently [15]. Any ambiguities on data charting would be discussed and resolved by the senior author.

\subsection{Extracted Data Items}

- Study identification Author, year of publication.

- Methods and inclusion criteria

Population (sample size, whether specific age range was stated and if yes, the actual age range). Existence of an inclusion criterion determining a minimum tinnitus duration reported by the patients or not.

Existence of an inclusion criterion determining whether patients reported tinnitus as a primary complain or not.

Consideration of hearing loss both in inclusion criteria (range and thresholds of hearing loss provided) as well as in data analysis and interpretation.

Whether a limitation in regards to tinnitus laterality was present or not (whether only unilateral or bilateral cases were included, whether both unilateral and bilateral were included or whether no relevant criterion was set).

- Methods and study procedures

The primary objective of the study.

The treatments applied in the intervention and the control group. 
Whether randomization procedure was properly and adequately described. Metric for this was the ability to reproduce the procedure based on the details provided.

Whether power analysis was presented (either ad hoc or post hoc) and the estimated power if available.

Follow up period duration and schedule of follow up visits.

- Methods and outcome measures

Outcome measures used, and whether a primary outcome measure was clearly defined.

- Results

Results in terms both of clinically and statistically significant improvement in each one of the groups as well as of difference between groups. An estimation of whether intervention was considered effective is provided, based on whether interventional group showed statistical and clinical improvement in the primary outcome measure compared to baseline and also a statistically significant difference was found, compared to the control arm, in the case no intervention is conducted (no placebo, sham, or waiting list).

\subsection{Risk of Bias in Individual Studies}

In view of the nature of our research question and the objectives of this systematic review, potential bias and methodological pitfalls of individual studies make part of the core analysis and results interpretation.

\subsection{Synthesis of Results}

We present the included studies and summarize the extracted data items in form of tables, with emphasis on our predefined scientific queries. Further analysis in plain text and interpretation of the results shall be found in the corresponding discussion sections.

\section{Results}

\subsection{Study Selection}

Seventy-three articles were identified. The screening procedure is reflected in Figure 1.

\subsection{Study Characteristics}

A detailed summary of the characteristics of the eligible studies can be found in the Tables 1 and 2 .

\subsection{Results of Individuals Studies}

\subsubsection{Methods and Inclusion Criteria}

Across studies, participants' screening and recruitment are focusing on participants' age and presence of tinnitus, while only a small proportion of studies use specific characteristics of tinnitus as inclusion criteria (Table 1). With regards to age, 20 studies set as participants' age upper limit the seventh decade of life, while 27 of them do not provide a clear report of this information. Seven studies have excluded participants for whom tinnitus was not their primary complaint, while there is a large heterogeneity among studies with regards to the tinnitus onset and minimum duration criterion; 2, 3, 6, and 12 months have all been used in the studies included in this review. In sixteen of them, no specification on time and/or duration is provided. Whether tinnitus is continuous or intermittent was not specified as an inclusion criterion in the investigated studies. Many studies defined a minimum annoyance level as inclusion criterion, measured either by means of simple visual analog scales (VAS) or through specific questionnaires (Tinnitus Functional Index (TFI), Tinnitus Handicap Index (THI), Tinnitus Handicap Questionnaire (THQ), and the Tinnitus Acceptance Questionnaire (TAQ)). Four studies excluded participants with hearing loss, while the grand majority of studies do not mention the hearing status of their sample. No study involving other than hearing aid fitting as study intervention had screened candidates for the use of hearing aids prior to their entry to the study. 
Table 1. Characteristics of included studies: Inclusion criteria.

\begin{tabular}{|c|c|c|c|c|c|c|c|c|c|}
\hline Author, Year & Primary Objective & $N$ & $\begin{array}{l}\text { Age } \\
\text { (Years) }\end{array}$ & $\begin{array}{l}\text { Tinnitus as } \\
\text { Primary } \\
\text { Complaint }\end{array}$ & $\begin{array}{l}\text { Tinnitus } \\
\text { Onset } \\
\text { (Months) }\end{array}$ & $\begin{array}{c}\text { Tinnitus } \\
\text { Laterality } \\
\text { Uni/Bilateral }\end{array}$ & $\begin{array}{l}\text { Minimum } \\
\text { Tinnitus } \\
\text { Threshold }\end{array}$ & Hearing Loss & $\begin{array}{l}\text { Use of } \\
\text { Hearing Aids } \\
\text { Considered }\end{array}$ \\
\hline $\begin{array}{l}\text { Anders, } 2010 \\
{[16]}\end{array}$ & $\begin{array}{l}\text { Evaluation of the efficacy of } 1 \mathrm{~Hz} \text { repetitive } \\
\text { transcranial magnetic stimulation (rTMS) in the } \\
\text { treatment of tinnitus. }\end{array}$ & 42 & $18-70$ & No & $>6$ months & Both & No & $\begin{array}{l}\text { Age-adjusted } \\
\text { normal } \\
\text { sensorineural } \\
\text { hearing }\end{array}$ & No \\
\hline $\begin{array}{c}\text { Biesinger, } 2010 \\
\text { [17] }\end{array}$ & $\begin{array}{l}\text { Effect of a Qigong intervention on patients with } \\
\text { tinnitus with somatosensoric components }\end{array}$ & 80 & & Yes & $>3$ months & $\begin{array}{c}\text { Not } \\
\text { determined }\end{array}$ & No & Normal audiogram & No \\
\hline $\begin{array}{l}\text { Dehkordi, } \\
2011[18]\end{array}$ & $\begin{array}{l}\text { Effect of gabapentin therapy on } \\
\text { idiopathic tinnitus }\end{array}$ & 80 & $18-85$ & No & $>2$ months & Unilateral & No & Not determined & No \\
\hline $\begin{array}{l}\text { Sziklai, } 2011 \\
\text { [19] }\end{array}$ & $\begin{array}{l}\text { Effect of pramipexole, a dopamine receptor } \\
\text { agonist, influenced tinnitus associated } \\
\text { with presbycusis }\end{array}$ & 40 & $>50$ & No & $>1$ year & $\begin{array}{c}\text { Not } \\
\text { determined }\end{array}$ & No & Bilateral SNHL & No \\
\hline $\begin{array}{l}\text { Westin, } 2011 \\
\text { [20] }\end{array}$ & $\begin{array}{l}\text { Comparison of acceptance and commitment } \\
\text { therapy (ACT) with tinnitus retraining therapy } \\
\text { (TRT) on tinnitus }\end{array}$ & 64 & $\geq 18$ & Yes & $\geq 6$ months & $\begin{array}{c}\text { Not } \\
\text { determined }\end{array}$ & $\mathrm{THI} \geq 30$ & $\begin{array}{l}\text { Hearing thresholds } \\
\text { which would allow } \\
\text { for the use of } \\
\text { wearable sound } \\
\text { generators }\end{array}$ & No \\
\hline $\begin{array}{l}\text { Cima, } 2012 \\
{[21]}\end{array}$ & $\begin{array}{c}\text { Effect of cognitive behavioral therapy (CBT) } \\
\text { versus usual care }\end{array}$ & 492 & $>18$ & No & & $\begin{array}{c}\text { Not } \\
\text { determined }\end{array}$ & No & Not determined & No \\
\hline Han, 2012 [22] & $\begin{array}{l}\text { Comparison between Clonazepam and } \\
\text { gingko biloba } \\
\end{array}$ & 38 & & No & 2 months & $\begin{array}{c}\text { Not } \\
\text { determined } \\
\end{array}$ & No & Not determined & No \\
\hline $\begin{array}{c}\text { Hesser, } 2012 \\
\text { [23] }\end{array}$ & $\begin{array}{c}\text { Effects on global tinnitus severity of } 2 \\
\text { Internet-delivered psychological treatments, } \\
\text { acceptance, and commitment therapy (ACT) and } \\
\text { CBT, in guided self-help format }\end{array}$ & 99 & $>18$ & No & $>6$ months & $\begin{array}{c}\text { Not } \\
\text { determined }\end{array}$ & $\mathrm{THI} \geq 38$ & Not determined & No \\
\hline $\begin{array}{c}\text { Hoare, } 2012 \\
{[24]}\end{array}$ & $\begin{array}{l}\text { Comparison between different methods of } \\
\text { frequency discrimination training on } \\
\text { tinnitus percept }\end{array}$ & 70 & & No & 6 months & $\begin{array}{c}\text { Not } \\
\text { determined }\end{array}$ & No & $\begin{array}{l}<40 \mathrm{~dB} \text { in at least } \\
\text { one frequency }\end{array}$ & No \\
\hline Jeon, 2012 [25] & Effect of acupuncture versus sham & 33 & $18-60$ & No & 6 months & Unilateral & No & $\begin{array}{l}\text { Mean of } 0.5,1 \text {, and } \\
\quad 2 \mathrm{kHz} \\
\text { Audiogram }>50 \mathrm{~dB}\end{array}$ & No \\
\hline
\end{tabular}


Table 1. Cont.

\begin{tabular}{|c|c|c|c|c|c|c|c|c|c|}
\hline Author, Year & Primary Objective & $N$ & $\begin{array}{c}\text { Age } \\
\text { (Years) }\end{array}$ & $\begin{array}{l}\text { Tinnitus as } \\
\text { Primary } \\
\text { Complaint }\end{array}$ & $\begin{array}{l}\text { Tinnitus } \\
\text { Onset } \\
\text { (Months) }\end{array}$ & $\begin{array}{c}\text { Tinnitus } \\
\text { Laterality } \\
\text { Uni/Bilateral }\end{array}$ & $\begin{array}{c}\text { Minimum } \\
\text { Tinnitus } \\
\text { Threshold }\end{array}$ & Hearing Loss & $\begin{array}{c}\text { Use of } \\
\text { Hearing Aids } \\
\text { Considered }\end{array}$ \\
\hline $\begin{array}{c}\text { Kreuzer, } 2012 \\
\text { [26] }\end{array}$ & $\begin{array}{l}\text { Efficacy of a specific mindfulness- and } \\
\text { body-psychotherapy based program in patients } \\
\text { suffering from chronic tinnitus }\end{array}$ & 36 & $18-80$ & No & $>6$ months & $\begin{array}{c}\text { Not } \\
\text { determined }\end{array}$ & No & Not determined & No \\
\hline $\begin{array}{l}\text { Ngao, } 2012 \\
\quad[27]\end{array}$ & $\begin{array}{c}\text { Effect of transmeatal low-power laser } \\
\text { stimulation (TLLS) }\end{array}$ & 43 & & Yes & & $\begin{array}{c}\text { Not } \\
\text { determined }\end{array}$ & No & Not determined & No \\
\hline $\begin{array}{c}\text { Rocha, } 2012 \\
\text { [29] }\end{array}$ & $\begin{array}{l}\text { Efficacy of myofascial trigger point (MTP) } \\
\text { deactivation for tinnitus relief in patients with } \\
\text { myofascial pain syndrome }\end{array}$ & 71 & & No & $>3$ months & $\begin{array}{c}\text { Not } \\
\text { determined }\end{array}$ & No & Not determined & No \\
\hline Tass, 2012 [30] & $\begin{array}{c}\text { Comparison between CR (4 different groups) } \\
\text { vs. placebo }\end{array}$ & 63 & $>18$ & No & 6 months & $\begin{array}{c}\text { Not } \\
\text { determined }\end{array}$ & No & $\begin{array}{l}<50 \mathrm{~dB} \text { in all } \\
\text { frequencies }\end{array}$ & No \\
\hline Choi, 2013 [31] & $\begin{array}{l}\text { Comparison between intratympanic steroids } \\
\text { and placebo }\end{array}$ & 30 & & No & & $\begin{array}{c}\text { Not } \\
\text { determined }\end{array}$ & No & Not determined & No \\
\hline $\begin{array}{c}\text { Coelho, } 2013 \\
\text { [32] }\end{array}$ & Effect of zinc versus placebo & 115 & $>60$ & No & 6 months & $\begin{array}{c}\text { Not } \\
\text { determined }\end{array}$ & No & Not determined & No \\
\hline $\begin{array}{c}\text { Hoekstra, } 2013 \\
{[33]}\end{array}$ & $\begin{array}{l}\text { Effect of repetitive transcranial magnetic } \\
\text { stimulation(rTMS) on tinnitus }\end{array}$ & 50 & & No & $>2$ months & $\begin{array}{c}\text { Not } \\
\text { determined }\end{array}$ & No & Not determined & No \\
\hline $\begin{array}{l}\text { Mollasadeghi, } \\
2013 \text { [34] }\end{array}$ & Effect of low laser beam in tinnitus & 89 & $\leq 50$ & No & & $\begin{array}{c}\text { Not } \\
\text { determined }\end{array}$ & No & $\begin{array}{c}>15 \mathrm{~dB} \text { at least at } \\
\text { one of } 3,4 \text {, and } \\
6 \mathrm{kHz}\end{array}$ & No \\
\hline $\begin{array}{l}\text { Nyenhuis, } \\
2013 \text { [35] }\end{array}$ & $\begin{array}{l}\text { The efficacy of CBT-oriented interventions for } \\
\text { acute tinnitus on a broader data basis. }\end{array}$ & 185 & $18-75$ & No & 2-26 weeks & $\begin{array}{c}\text { Not } \\
\text { determined }\end{array}$ & No & Not determined & No \\
\hline $\begin{array}{l}\text { Sönmez, } 2013 \\
{[36]}\end{array}$ & Comparison between ozone and betahistine & 68 & $18-75$ & No & 6 months & $\begin{array}{c}\text { Not } \\
\text { determined }\end{array}$ & No & No & No \\
\hline $\begin{array}{c}\text { Taslimi, } 2013 \\
\text { [37] }\end{array}$ & Effect of ondansedron & 53 & $18-70$ & No & 3 months & $\begin{array}{c}\text { Not } \\
\text { determined }\end{array}$ & No & Not determined & No \\
\hline
\end{tabular}


Table 1. Cont.

\begin{tabular}{|c|c|c|c|c|c|c|c|c|c|}
\hline Author, Year & Primary Objective & $N$ & $\begin{array}{l}\text { Age } \\
\text { (Years) }\end{array}$ & $\begin{array}{l}\text { Tinnitus as } \\
\text { Primary } \\
\text { Complaint }\end{array}$ & $\begin{array}{l}\text { Tinnitus } \\
\text { Onset } \\
\text { (Months) }\end{array}$ & $\begin{array}{c}\text { Tinnitus } \\
\text { Laterality } \\
\text { Uni/Bilateral }\end{array}$ & $\begin{array}{l}\text { Minimum } \\
\text { Tinnitus } \\
\text { Threshold }\end{array}$ & Hearing Loss & $\begin{array}{c}\text { Use of } \\
\text { Hearing Aids } \\
\text { Considered }\end{array}$ \\
\hline $\begin{array}{l}\text { Dos Santos, } \\
2014[38]\end{array}$ & $\begin{array}{l}\text { Evaluation of combined use of amplification and } \\
\text { sound generator and their combination }\end{array}$ & 49 & & No & $\begin{array}{l}\text { At least } \\
6 \text { months }\end{array}$ & $\begin{array}{l}\text { Not } \\
\text { determined }\end{array}$ & THI $>20$ & $\begin{array}{l}\text { Mild to moderate } \\
\text { symmetrical } \\
\text { sensorineural hl }\end{array}$ & No \\
\hline $\begin{array}{l}\text { Hoare, } 2014 \\
\text { [39] }\end{array}$ & $\begin{array}{c}\text { Frequency discrimination training (FDT) } \\
\text { delivered in a gaming format have significant } \\
\text { therapeutic benefit in tinnitus }\end{array}$ & 60 & & No & & $\begin{array}{c}\text { Not } \\
\text { determined }\end{array}$ & No & $\begin{array}{l}\geq 20 \mathrm{~dB} \text { in at least } \\
\text { one frequency, } \\
\leq 40 \mathrm{~dB} \text { average }\end{array}$ & No \\
\hline $\begin{array}{c}\text { Jasper, } 2014 \\
\text { [40] }\end{array}$ & $\begin{array}{l}\text { Effects of conventional face-to-face group } \\
\text { cognitive behavioral therapy (GCBT) and an } \\
\text { Internet-delivered guided self-help treatment } \\
\text { iCBT on tinnitus distress }\end{array}$ & 128 & $\geq 18$ & Yes & $\geq 6$ months & $\begin{array}{c}\text { Not } \\
\text { determined }\end{array}$ & $\begin{array}{l}\mathrm{THI} \geq 18 \text { or } \\
\text { mini-TQ } \geq 8\end{array}$ & Not determined & No \\
\hline $\begin{array}{l}\text { Shekhawat, } \\
2014 \text { [41] }\end{array}$ & $\begin{array}{l}\text { Comparison of multisession anodal transcranial } \\
\text { direct current stimulation (TDCS) of the left } \\
\text { temporoparietal area would enhance sound } \\
\text { therapy from hearing aids. }\end{array}$ & 40 & & No & $>2$ years & $\begin{array}{c}\text { Not } \\
\text { determined }\end{array}$ & $\mathrm{TFI}>25$ & Aidable HL & No \\
\hline $\begin{array}{l}\text { Dehkordi, } \\
2015[43]\end{array}$ & $\begin{array}{l}\text { Effect of low-dose laser therapy on chronic } \\
\text { cochlear tinnitus }\end{array}$ & 66 & & No & & $\begin{array}{c}\text { Not } \\
\text { determined }\end{array}$ & No & Not mentioned & No \\
\hline $\begin{array}{c}\text { Bilici, } 2015 \\
\quad[44]\end{array}$ & 5 groups: 3 types of rTMS, paroxetine, placebo & 75 & & No & 1 year & $\begin{array}{c}\text { Not } \\
\text { determined }\end{array}$ & No & Normal hearing & No \\
\hline $\begin{array}{l}\text { Folmer, } 2015 \\
{[45]}\end{array}$ & $\begin{array}{l}\text { Effect of repeated transcranial } \\
\text { magnetic stimulation }\end{array}$ & 61 & & No & & $\begin{array}{c}\text { Not } \\
\text { determined }\end{array}$ & No & Not determined & No \\
\hline $\begin{array}{l}\text { Kreuzer, } 2015 \\
{[46]}\end{array}$ & $\begin{array}{l}\text { Comparison of medial frontal stimulation with } \\
\text { double cone coil and conventional prefrontal left } \\
\text { dorsolateral prefrontal cortex } \\
\text { (DLPFC)-stimulation (study arm } 2 / \text { control } \\
\text { group) both followed by stimulation of the left } \\
\text { temporo-parietal junction area }\end{array}$ & 40 & & No & $>6$ months & $\begin{array}{c}\text { Not } \\
\text { determined }\end{array}$ & No & Not determined & No \\
\hline
\end{tabular}


Table 1. Cont.

\begin{tabular}{|c|c|c|c|c|c|c|c|c|c|}
\hline Author, Year & Primary Objective & $N$ & $\begin{array}{l}\text { Age } \\
\text { (Years) }\end{array}$ & $\begin{array}{l}\text { Tinnitus as } \\
\text { Primary } \\
\text { Complaint }\end{array}$ & $\begin{array}{l}\text { Tinnitus } \\
\text { Onset } \\
\text { (Months) }\end{array}$ & $\begin{array}{c}\text { Tinnitus } \\
\text { Laterality } \\
\text { Uni/Bilateral }\end{array}$ & $\begin{array}{l}\text { Minimum } \\
\text { Tinnitus } \\
\text { Threshold }\end{array}$ & Hearing Loss & $\begin{array}{l}\text { Use of } \\
\text { Hearing Aids } \\
\text { Considered }\end{array}$ \\
\hline $\begin{array}{l}\text { Malinvaud, } \\
2015 \text { [47] }\end{array}$ & $\begin{array}{c}\text { Comparison between CBT and virtual reality } \\
\text { interactive intervention }\end{array}$ & 148 & $18-70$ & No & 12 months & Unilateral & No & Normal to mild & No \\
\hline Pal, 2015 [48] & $\begin{array}{l}\text { Investigation of the efficacy and safety of } \\
\text { repeated sessions of a novel transcranial direct } \\
\text { current stimulation (TDCS) protocol by } \\
\text { combining bilateral cathodal TDCS to the } \\
\text { auditory cortex (AC) with anodal stimulation of } \\
\text { the prefrontal cortex (PFC). }\end{array}$ & 42 & & No & $\geq 1$ year & $\begin{array}{c}\text { Not } \\
\text { determined }\end{array}$ & No & $\begin{array}{l}\text { Age-adjusted } \\
\text { normal hearing } \\
\text { according to the } \\
\text { presbycusis scale }\end{array}$ & No \\
\hline $\begin{array}{c}\text { Thabit, } 2015 \\
\text { [49] }\end{array}$ & $\begin{array}{l}\text { Effect of different types of rTMS and } \\
\text { their combination }\end{array}$ & 30 & $>18$ & No & 6 months & Both & No & Not determined & No \\
\hline $\begin{array}{l}\text { Albu, } 2016 \\
\quad[50]\end{array}$ & $\begin{array}{c}\text { Effectiveness of intratympanic (IT) steroids and } \\
\text { melatonine versus melatonine only in } \\
\text { acute tinnitus }\end{array}$ & 60 & & No & $\begin{array}{l}\text { Acute (within } \\
3 \text { months) }\end{array}$ & Unilateral & No & Not mentioned & No \\
\hline Doi, 2016 [51] & Effectiveness of acupuncture therapy for tinnitus & 50 & & No & $\begin{array}{c}\text { Not } \\
\text { determined }\end{array}$ & $\begin{array}{c}\text { Not } \\
\text { determined }\end{array}$ & $\begin{array}{l}\text { THI: } \\
\text { moderate to } \\
\text { severe }\end{array}$ & Not determined & No \\
\hline $\begin{array}{c}\text { Henry, } 2016 \\
{[52]}\end{array}$ & Effect on tinnitus severity by using tm-TRT-ted & 148 & & No & & $\begin{array}{c}\text { Not } \\
\text { determined }\end{array}$ & No & Not determined & No \\
\hline $\begin{array}{l}\text { Laureano, } \\
2016[53]\end{array}$ & $\begin{array}{l}\text { Effect of acupuncture on brain perfusion using } \\
\text { (99m) ethyl cysteinate dimer single-photon } \\
\text { emission computed tomography ( }(99 \mathrm{~m}) \text { Tc-ECD } \\
\text { SPECT) in patients with tinnitus }\end{array}$ & 57 & $18-60$ & No & $>3$ months & Both & No & $\mathrm{Up}$ to $25 \mathrm{~dB}$ & No \\
\hline $\begin{array}{c}\text { Lehner, } 2016 \\
{[54]}\end{array}$ & Comparison between two types of rTMS & 49 & $18-70$ & No & 6 months & $\begin{array}{c}\text { Not } \\
\text { determined }\end{array}$ & $\mathrm{THI}>38$ & Not determined & No \\
\hline Li, 2016 [55] & $\begin{array}{l}\text { Compare the effects of personalized, altered } \\
\text { music to unaltered music on subjective tinnitus }\end{array}$ & 34 & $\geq 18$ & No & $\geq 12$ months & Both & $\mathrm{THI}>26$ & $\begin{array}{l}\text { Hearing loss } \\
\leq 70 \mathrm{~dB}\end{array}$ & No \\
\hline Lim, $2016[56]$ & $\begin{array}{c}\text { Efficacy of cilostazol, a selective } \\
\text { phosphodiesterase } 3 \text { inhibitor, in patients with } \\
\text { chronic tinnitus }\end{array}$ & 50 & $>19$ & No & $3-12$ months & Both & Vas $\geq 4$ & Not determined & No \\
\hline
\end{tabular}


Table 1. Cont.

\begin{tabular}{|c|c|c|c|c|c|c|c|c|c|}
\hline Author, Year & Primary Objective & $N$ & $\begin{array}{l}\text { Age } \\
\text { (Years) }\end{array}$ & $\begin{array}{l}\text { Tinnitus as } \\
\text { Primary } \\
\text { Complaint }\end{array}$ & $\begin{array}{l}\text { Tinnitus } \\
\text { Onset } \\
\text { (Months) }\end{array}$ & $\begin{array}{c}\text { Tinnitus } \\
\text { Laterality } \\
\text { Uni/Bilateral }\end{array}$ & $\begin{array}{c}\text { Minimum } \\
\text { Tinnitus } \\
\text { Threshold }\end{array}$ & Hearing Loss & $\begin{array}{c}\text { Use of } \\
\text { Hearing Aids } \\
\text { Considered }\end{array}$ \\
\hline $\begin{array}{l}\text { Rojas- } \\
\text { roncancio, } \\
2016[57]\end{array}$ & $\begin{array}{l}\text { Effect of manganese and lipoflavonoid plus } \\
\text { on tinnitus }\end{array}$ & 40 & & No & $>6$ months & $\begin{array}{l}\text { Not } \\
\text { determined }\end{array}$ & $\begin{array}{c}\text { Tinnitus } \\
\text { loudness and } \\
\text { annoyance }> \\
50 \%\end{array}$ & Not determined & No \\
\hline $\begin{array}{c}\text { Roland, } 2016 \\
{[58]}\end{array}$ & $\begin{array}{l}\text { Evaluation of the neural network changes in } \\
\text { patients with bothersome chronic tinnitus who } \\
\text { underwent rTMS treatment targeting the left } \\
\text { temporoparietal junction (TPJ), as compared to } \\
\text { those who received sham therapy. }\end{array}$ & 30 & $18-60$ & No & $\geq 6$ months & $\begin{array}{c}\text { Not } \\
\text { determined }\end{array}$ & $\mathrm{THI}>30$ & Not determined & No \\
\hline $\begin{array}{c}\text { Singh, } 2016 \\
\text { [59] }\end{array}$ & Effect of B12 versus placebo & 40 & $18-60$ & No & 6 months & $\begin{array}{c}\text { Not } \\
\text { determined }\end{array}$ & No & Not determined & No \\
\hline $\begin{array}{l}\text { Stein, } 2016 \\
\quad[60]\end{array}$ & $\begin{array}{c}\text { The effect of a sound therapy (tailor-made } \\
\text { notched music training, TMNMT) } \\
\text { against tinnitus }\end{array}$ & 100 & $18-70$ & No & $\geq 3$ months & Both & No & $\begin{array}{l}\mathrm{Hl} \leq 70 \mathrm{~dB} \text { hl in the } \\
\text { frequency ranges of } \\
\text { one-half octave } \\
\text { above and below } \\
\text { the tinnitus } \\
\text { frequency }\end{array}$ & No \\
\hline $\begin{array}{c}\text { Weise, } 2016 \\
\text { [61] }\end{array}$ & Effect of iCBT & 61 & $>18$ & Yes & $>6$ months & $\begin{array}{c}\text { Not } \\
\text { determined }\end{array}$ & $\begin{array}{c}\mathrm{THI}>38 \text { or } \\
\text { mini-TQ }>13\end{array}$ & Not determined & No \\
\hline Wise, 2016 [62] & $\begin{array}{l}\text { Effects of an auditory attention training game } \\
\text { with those of a control game across tinnitus, } \\
\text { attention, and electrophysiological measures }\end{array}$ & 31 & $18-70$ & No & $>6$ months & $\begin{array}{l}\text { Not } \\
\text { determined }\end{array}$ & $\begin{array}{l}\text { Tinnitus } \\
\text { problem } \\
\text { rating scale > } \\
\text { mild }\end{array}$ & $\begin{array}{c}<80 \mathrm{Db} \mathrm{HL} \\
\text { nonconductive HL }\end{array}$ & No \\
\hline $\begin{array}{l}\text { Zarenoe, } 2016 \\
{[63]}\end{array}$ & $\begin{array}{l}\text { Effects of motivational interview (MI) as an } \\
\text { adjunct to regular HA fitting for patients with } \\
\text { tinnitus and hearing loss. }\end{array}$ & 46 & & No & & $\begin{array}{c}\text { Not } \\
\text { determined }\end{array}$ & No & Not determined & No \\
\hline $\begin{array}{c}\text { Elzayat, } 2016 \\
{[64]}\end{array}$ & $\begin{array}{l}\text { To evaluate the effectiveness of adding lidocaine } \\
\text { to intratympanic steroid in the patients with } \\
\text { idiopathic subjective tinnitus (IST). }\end{array}$ & 44 & & No & & $\begin{array}{l}\text { Not } \\
\text { determined }\end{array}$ & No & Not determined & No \\
\hline $\begin{array}{l}\text { Kallogjeri, } \\
2017[65]\end{array}$ & $\begin{array}{l}\text { To evaluate the effect of the brain fitting } \\
\text { program-tinnitus on tinnitus. }\end{array}$ & 60 & $20-65$ & No & $>6$ months & $\begin{array}{c}\text { Not } \\
\text { determined }\end{array}$ & $\begin{array}{l}\text { According to } \\
\text { bothersome } \\
\text { scale }\end{array}$ & Not determined & No \\
\hline
\end{tabular}


Table 1. Cont.

\begin{tabular}{|c|c|c|c|c|c|c|c|c|c|}
\hline Author, Year & Primary Objective & $N$ & $\begin{array}{c}\text { Age } \\
\text { (Years) }\end{array}$ & $\begin{array}{l}\text { Tinnitus as } \\
\text { Primary } \\
\text { Complaint }\end{array}$ & $\begin{array}{l}\text { Tinnitus } \\
\text { Onset } \\
\text { (Months) }\end{array}$ & $\begin{array}{c}\text { Tinnitus } \\
\text { Laterality } \\
\text { Uni/Bilateral }\end{array}$ & $\begin{array}{l}\text { Minimum } \\
\text { Tinnitus } \\
\text { Threshold }\end{array}$ & Hearing Loss & $\begin{array}{c}\text { Use of } \\
\text { Hearing Aids } \\
\text { Considered }\end{array}$ \\
\hline Kim, 2017 [66] & Effect of different approaches of acupuncture & 39 & $20-75$ & No & 2 weeks & $\begin{array}{c}\text { Not } \\
\text { determined }\end{array}$ & No & Not determined & No \\
\hline $\begin{array}{l}\text { Landgrebe, } \\
2017[67]\end{array}$ & $\begin{array}{l}\text { Evaluation of the efficacy of a two-week } \\
\text { 1-Hz-RTMS in patients with chronic tinnitus. }\end{array}$ & 146 & $18-70$ & No & $>6$ months & $\begin{array}{c}\text { Not } \\
\text { determined }\end{array}$ & $\mathrm{THI}>38$ & $\begin{array}{l}\text { Normal, } \\
\text { age-adjusted } \\
\text { hearing levels. } \\
\text { Conductive hearing } \\
\text { loss } \leq 15 \mathrm{db} .\end{array}$ & No \\
\hline $\begin{array}{l}\text { Mckenna, } 2017 \\
{[68]}\end{array}$ & $\begin{array}{l}\text { Effect of mindfulness based cognitive therapy } \\
(\mathrm{MBCT}) \text { in tinnitus severity, psychological } \\
\text { distress, functional disability, avoidance, and } \\
\text { negative cognitions and a greater increase in } \\
\text { tinnitus acceptance. }\end{array}$ & 75 & $\geq 18$ & No & $>6$ months & $\begin{array}{c}\text { Not } \\
\text { determined }\end{array}$ & No & $\begin{array}{l}\text { Hearing levels } \\
\text { allowing } \\
\text { participation in } \\
\text { group discussions }\end{array}$ & No \\
\hline Arif, 2017 [69] & Relaxation therapy and mindfulness & 61 & $>18$ & Yes & & $\begin{array}{c}\text { Not } \\
\text { determined }\end{array}$ & No & Not determined & No \\
\hline $\begin{array}{l}\text { Sahlsten, } 2017 \\
{[71]}\end{array}$ & $\begin{array}{l}\text { E-field navigation should versus } \\
\text { non-navigated rTMS }\end{array}$ & 39 & $18-65$ & No & $\begin{array}{l}6 \text { months-10 } \\
\text { years }\end{array}$ & Both & No & Not determined & No \\
\hline $\begin{array}{l}\text { Theodoroff, } \\
2017 \text { [72] }\end{array}$ & $\begin{array}{l}\text { To determine if an acoustic stimulus mimicking } \\
\text { the tinnitus perception delivered during sleep } \\
\text { from the Otoharmonics corporation's LEVO } \\
\text { system reduces tinnitus-related distress and/or } \\
\text { perceived loudness of tinnitus during awake } \\
\text { hours for people who experience } \\
\text { bothersome tinnitus }\end{array}$ & 58 & $30-72$ & No & $>6$ months & $\begin{array}{c}\text { Not } \\
\text { determined }\end{array}$ & TFI $>25$ & $\begin{array}{l}<70 \mathrm{~dB} \text { hl, in all } \\
\text { frequencies between } \\
0.25 \text { and } 8 \mathrm{kHz}\end{array}$ & No \\
\hline Tyler, 2017 [73] & $\begin{array}{l}\text { Effect of vagus nerve stimulation (VNS) paired } \\
\text { with sounds in chronic tinnitus patients }\end{array}$ & 30 & $22-65$ & No & $>1$ year & Both & No & Not determined & No \\
\hline Lee, 2018 [74] & Effect of intratympanic steroids on acute tinnitus & 54 & & No & $\begin{array}{c}\text { Acute } \\
\text { (one month) }\end{array}$ & Unilateral & No & Not determined & No \\
\hline
\end{tabular}


Table 1. Cont.

\begin{tabular}{|c|c|c|c|c|c|c|c|c|c|}
\hline Author, Year & Primary Objective & $N$ & $\begin{array}{l}\text { Age } \\
\text { (Years) }\end{array}$ & $\begin{array}{l}\text { Tinnitus as } \\
\text { Primary } \\
\text { Complaint }\end{array}$ & $\begin{array}{l}\text { Tinnitus } \\
\text { Onset } \\
\text { (Months) }\end{array}$ & $\begin{array}{c}\text { Tinnitus } \\
\text { Laterality } \\
\text { Uni/Bilateral }\end{array}$ & $\begin{array}{l}\text { Minimum } \\
\text { Tinnitus } \\
\text { Threshold }\end{array}$ & Hearing Loss & $\begin{array}{l}\text { Use of } \\
\text { Hearing Aids } \\
\text { Considered }\end{array}$ \\
\hline $\begin{array}{l}\text { Beukes, } 2018 \\
{[75]}\end{array}$ & $\begin{array}{l}\text { Evaluation of an Internet-based cognitive } \\
\text { behavioral therapy intervention versus face } \\
\text { to face }\end{array}$ & 92 & $>18$ & No & $\begin{array}{l}\text { Not } \\
\text { determined }\end{array}$ & $\begin{array}{c}\text { Not } \\
\text { determined }\end{array}$ & No & Not determined & Yes \\
\hline $\begin{array}{c}\text { Abtahi, } 2018 \\
{[76]}\end{array}$ & $\begin{array}{l}\text { Effectiveness of anodal and cathodal methods in } \\
\text { reducing the intensity of tinnitus }\end{array}$ & 51 & $18-80$ & No & $>1$ year & $\begin{array}{c}\text { Not } \\
\text { determined }\end{array}$ & No & Not determined & No \\
\hline $\begin{array}{l}\text { Hong, } 2018 \\
{[78]}\end{array}$ & Effect of nitrous oxide on tinnitus & & $18-65$ & No & $>6$ months & $\begin{array}{c}\text { Not } \\
\text { determined }\end{array}$ & $\begin{array}{l}\text { According to } \\
\text { bothersome } \\
\text { scale }\end{array}$ & Not determined & No \\
\hline $\begin{array}{l}\text { Godbehere, } \\
2019 \text { [79] }\end{array}$ & $\begin{array}{c}\text { Theta burst TMS are an effective treatment for } \\
\text { chronic tinnitus }\end{array}$ & 40 & $>18$ & No & $\begin{array}{c}\text { Not } \\
\text { determined }\end{array}$ & Both & No & $\begin{array}{l}\text { No HL, mild and } \\
\text { moderate HL }\end{array}$ & No \\
\hline Hall, 2019 [80] & $\begin{array}{c}\text { Effect of AUTt00063, a novel centrally acting } \\
\text { drug) potent and selective modulator of kv3.1 } \\
\text { and kv3.2 voltage-gated potassium channels) } \\
\text { vs. placebo }\end{array}$ & 76 & $>18$ & Yes & $\begin{array}{l}>6 \\
<18 \text { months }\end{array}$ & Both & $\begin{aligned} \text { TFI } & >24 \text { and } \\
& <68\end{aligned}$ & $\begin{array}{c}<60 \mathrm{db} \text { in } \\
0.5,1,2,4 \mathrm{kHz}\end{array}$ & No \\
\hline Li, 2019 [81] & $\begin{array}{l}\text { Clinical efficacy of cognitive behavioral therapy } \\
\text { (CBT) for treatment of chronic subjective tinnitus }\end{array}$ & 100 & & No & $>3$ months & $\begin{array}{c}\text { Not } \\
\text { determined }\end{array}$ & No & Not determined & No \\
\hline Noh, 2019 [82] & $\begin{array}{c}\text { To investigate the effects of active dual-site rTMS } \\
\text { treatment on reducing tinnitus using a } \\
\text { double-blind randomized controlled trial. }\end{array}$ & 30 & & No & & $\begin{array}{c}\text { Not } \\
\text { determined }\end{array}$ & No & Not determined & No \\
\hline $\begin{array}{l}\text { Prozchazkova, } \\
2019 \text { [83] }\end{array}$ & $\begin{array}{l}\text { Comparison between gingko biloba } \\
\text { and pentoxifylline }\end{array}$ & 197 & $>30$ & No & 3 months & $\begin{array}{c}\text { Not } \\
\text { determined }\end{array}$ & Mini TQ > 5 & Not determined & No \\
\hline $\begin{array}{l}\text { Radunz, } 2019 \\
{[84]}\end{array}$ & $\begin{array}{l}\text { Comparison between ginkgo biloba, } \mathrm{HA} \text {, and } \\
\text { their combination }\end{array}$ & 35 & $>18$ & No & 3 months & Both & No & $\begin{array}{l}\text { All types of hearing } \\
\text { loss }\end{array}$ & No \\
\hline $\begin{array}{l}\text { Sahlsten, } 2019 \\
\text { [85] }\end{array}$ & $\begin{array}{l}\text { Comparison of neuronavigated versus } \\
\text { non-navigated repetitive transcranial } \\
\text { magnetic stimulation }\end{array}$ & 40 & $18-65$ & No & $\begin{array}{l}6 \text { months-10 } \\
\text { years }\end{array}$ & Both & $\begin{array}{l}\text { Numeric } \\
\text { scale }>4\end{array}$ & Not determined & No \\
\hline
\end{tabular}


Table 1. Cont.

\begin{tabular}{|c|c|c|c|c|c|c|c|c|c|}
\hline Author, Year & Primary Objective & $N$ & $\begin{array}{l}\text { Age } \\
\text { (Years) }\end{array}$ & $\begin{array}{l}\text { Tinnitus as } \\
\text { Primary } \\
\text { Complaint }\end{array}$ & $\begin{array}{l}\text { Tinnitus } \\
\text { Onset } \\
\text { (Months) }\end{array}$ & $\begin{array}{c}\text { Tinnitus } \\
\text { Laterality } \\
\text { Uni/Bilateral }\end{array}$ & $\begin{array}{l}\text { Minimum } \\
\text { Tinnitus } \\
\text { Threshold }\end{array}$ & Hearing Loss & $\begin{array}{c}\text { Use of } \\
\text { Hearing Aids } \\
\text { Considered }\end{array}$ \\
\hline $\begin{array}{c}\text { Scherer, } 2019 \\
{[86]}\end{array}$ & $\begin{array}{l}\text { To compare the efficacy of tinnitus retraining } \\
\text { therapy (TRT) and its components, ST, and TC, } \\
\text { with the standard of care (SOC) in reducing the } \\
\text { negative effect of tinnitus on quality of life. }\end{array}$ & 98 & & No & $>1$ year & $\begin{array}{c}\text { Not } \\
\text { determined }\end{array}$ & $\mathrm{TQ}>40$ & $\begin{array}{c}\text { Functionally } \\
\text { adequate hearing } \\
\text { sensitivity without } \\
\text { requirement of } \\
\text { amplification }\end{array}$ & No \\
\hline $\begin{array}{l}\text { Yakunina, } \\
2019 \text { [87] }\end{array}$ & $\begin{array}{l}\text { Evaluation of the effects on tinnitus of hearing } \\
\text { aids (HA) alone without accompanying } \\
\text { counseling or any other therapy additionally, } \\
\text { whether FL techniques (LFT and FT) performed } \\
\text { compared with conventional WDRC in the same } \\
\text { open-fit HA in terms of tinnitus suppression for } \\
\text { patients with high frequency hearing } \\
\text { loss (HFHL). }\end{array}$ & 94 & $>18$ & No & $\geq 3$ months & $\begin{array}{c}\text { Not } \\
\text { determined }\end{array}$ & $\begin{array}{c}\mathrm{THI}>18 \\
\text { Vas } \geq 50 \%\end{array}$ & SNHL & No \\
\hline $\begin{array}{l}\text { Tutar, } 2020 \\
{[88]}\end{array}$ & $\begin{array}{l}\text { Efficacy of transcutaneous electric stimulation } \\
\text { applied to the auricula }\end{array}$ & 60 & $18-65$ & No & $>3$ months & $\begin{array}{c}\text { Not } \\
\text { determined }\end{array}$ & No & Not determined & No \\
\hline
\end{tabular}

Table 2. Characteristics of included studies: study procedures and outcome measures.

\begin{tabular}{|c|c|c|c|c|c|c|c|c|}
\hline $\begin{array}{l}\text { Author, } \\
\text { Year }\end{array}$ & Treatment & $\begin{array}{l}\text { Control Group } \\
\text { Intervention }\end{array}$ & Randomization & Outcome Measures & $\begin{array}{l}\text { Monitoring } \\
\text { Duration }\end{array}$ & $\begin{array}{c}\text { Power } \\
\text { Analysis }\end{array}$ & Results & $\begin{array}{l}\text { Is Treatment } \\
\text { Effective? }\end{array}$ \\
\hline $\begin{array}{l}\text { Abtahi, } \\
2018[76]\end{array}$ & $\begin{array}{l}\text { Anodal Stimulation, } \\
\text { Cathodal Stimulation }\end{array}$ & Sham Stimulation & Unclear & $\begin{array}{c}\text { Tinnitus Intensity } \\
\text { Variations on A Scale } \\
\text { Between }-4 \text { and }+4 . \text { In This } \\
\text { Scale, }-4 \text { Indicated } \\
\text { Worsening Conditions, }+4 \\
\text { Meant Full Recovery, And } \\
\text { Zero Conveyed No Change } \\
\text { in The Tinnitus Intensity. }\end{array}$ & 2 Months & No & $\begin{array}{c}\text { Anodal Stimulation Was More } \\
\text { Effective Than the Cathodal and } \\
\text { Control Stimulation in Reducing the } \\
\text { Intensity of Tinnitus in The } \\
\text { Short Term }\end{array}$ & $\begin{array}{c}\text { Yes, Between } \\
\text { Two Versions } \\
\text { of The Same } \\
\text { Treatment }\end{array}$ \\
\hline $\begin{array}{c}\text { Albu, } \\
2016 \text { [50] }\end{array}$ & $\begin{array}{l}\text { Intratympanic (IT) } \\
\text { Steroid and } \\
\text { Melatonin }\end{array}$ & Melatonin & Unclear & THI, PSQI, BDI & 3 Months & No & $\begin{array}{c}\text { Better Response in The Combined } \\
\text { Group of Melatonine And IT In Acute } \\
\text { Tinnitus Patients }\end{array}$ & Yes \\
\hline
\end{tabular}


Table 2. Cont.

\begin{tabular}{|c|c|c|c|c|c|c|c|c|}
\hline $\begin{array}{l}\text { Author, } \\
\text { Year }\end{array}$ & Treatment & $\begin{array}{l}\text { Control Group } \\
\text { Intervention }\end{array}$ & Randomization & Outcome Measures & $\begin{array}{l}\text { Monitoring } \\
\text { Duration }\end{array}$ & $\begin{array}{l}\text { Power } \\
\text { Analysis }\end{array}$ & Results & $\begin{array}{l}\text { Is Treatment } \\
\text { Effective? }\end{array}$ \\
\hline $\begin{array}{l}\text { Anders, } \\
2010[16]\end{array}$ & $\begin{array}{l}\text { Active or Sham } \\
\text { repetitive } \\
\text { transcranial magnetic } \\
\text { stimulation (rTMS) }\end{array}$ & Sham Rtms & Unclear & VAS, THI & 26 Weeks & No & $\begin{array}{l}1 \mathrm{~Hz} \text { Rtms Treatment Was Capable of } \\
\text { Significantly Reducing the Total } \\
\text { Baseline Score of Basic Scales That } \\
\text { Measure Tinnitus Severity }\end{array}$ & No \\
\hline $\begin{array}{l}\text { Arif, } \\
2017[69]\end{array}$ & $\begin{array}{l}\text { Relaxation Therapy } \\
\text { or Mindfulness } \\
\text { Meditation } \\
\text { Treatment Over A } \\
\text { Period Of } 15 \text { Weeks }\end{array}$ & $\begin{array}{l}\text { Relaxation } \\
\text { Procedure }\end{array}$ & Clear & $\begin{array}{c}\text { Primary: TRQ } \\
\text { Secondary VAS and A } \\
\text { Health State Thermometer. }\end{array}$ & 15 Weeks & No & $\begin{array}{l}\text { Changes in Tinnitus Loudness and } \\
\text { THI (but not TRQ) with the } \\
\text { Customized Sound Therapy Were } \\
\text { Statistically Greater Than Those of } \\
\text { The Broadband Noise Therapy }\end{array}$ & No \\
\hline $\begin{array}{l}\text { Beukes, } \\
2017[70]\end{array}$ & $\begin{array}{l}\text { Internet-based } \\
\text { cognitive behavioral } \\
\text { treatment (iCBT) } \\
\text { Intervention }\end{array}$ & $\begin{array}{l}\text { ICBT After } \\
8 \text { Weeks }\end{array}$ & $\begin{array}{l}\text { Algorithm } \\
\text { Implemented by } \\
\text { Independent } \\
\text { Researcher }\end{array}$ & $\begin{array}{c}\text { Primary: TFI, Secondary: } \\
\text { ISI, GAD-7, PHQ-9, } \\
\text { HHIA-S, HQ, CFQ, SWLS }\end{array}$ & 2 Months & $80 \%$ & $\begin{array}{l}\text { Guided ICBT For Tinnitus Using } \\
\text { Audiological Support Resulted in } \\
\text { Statistically Significant Reductions in } \\
\text { Tinnitus Distress and Comorbidities } \\
\text { (Insomnia, Depression, Hyperacusis, } \\
\text { Cognitive Failures) And Improved } \\
\text { Quality of Life. }\end{array}$ & Yes \\
\hline $\begin{array}{l}\text { Biesinger, } \\
2010[17]\end{array}$ & $\begin{array}{l}10 \text { Qigong Training } \\
\text { Sessions }\end{array}$ & No Treatment & Unclear & VAS, TBF-12 & 3 Months & No & $\begin{array}{l}\text { No Statistically Significant Changes in } \\
\text { Both Groups }\end{array}$ & No \\
\hline $\begin{array}{l}\text { Bilici, } \\
2013[44]\end{array}$ & rTMS & $\begin{array}{l}\text { Paroxetine, } \\
\text { Placebo }\end{array}$ & Unclear & THI, TSI, BAS, PSS & 6 Months & No & $\begin{array}{l}\text { No Significant Improvement Neither } \\
\text { for Rtms Groups nor For Controls }\end{array}$ & No \\
\hline $\begin{array}{c}\text { Choi, } \\
2013[31]\end{array}$ & IT Steroids & Placebo & Clear & THI. VAS & 1 Month & No & $\begin{array}{l}\text { No Significant Difference Between IT } \\
\text { Steroids and Placebo }\end{array}$ & No \\
\hline $\begin{array}{l}\text { Cima, } \\
2012[21]\end{array}$ & CBT & Usual Care & Clear & HUI, HADS, TFQ & 12 Months & No & Superiority Of CBT & Yes \\
\hline $\begin{array}{l}\text { Coelho, } \\
2013 \text { [32] }\end{array}$ & Zinc & Placebo & Unclear & THQ & 4 Months & $90 \%$ & $\begin{array}{c}\text { No Significant Differences Between } \\
\text { Zinc and Placebo }\end{array}$ & No \\
\hline $\begin{array}{l}\text { Dehkordi, } \\
2011[18]\end{array}$ & Gabapentin & Placebo & Unclear & TSI & 26 Months & No & $\begin{array}{l}\text { No Statistically Significant Difference } \\
\text { Between the Two Groups In TSI. }\end{array}$ & No \\
\hline
\end{tabular}


Table 2. Cont.

\begin{tabular}{|c|c|c|c|c|c|c|c|c|}
\hline $\begin{array}{l}\text { Author, } \\
\text { Year }\end{array}$ & Treatment & $\begin{array}{l}\text { Control Group } \\
\text { Intervention }\end{array}$ & Randomization & Outcome Measures & $\begin{array}{l}\text { Monitoring } \\
\text { Duration }\end{array}$ & $\begin{array}{l}\text { Power } \\
\text { Analysis }\end{array}$ & Results & $\begin{array}{c}\text { Is Treatment } \\
\text { Effective? }\end{array}$ \\
\hline $\begin{array}{l}\text { Dehkordi, } \\
2015 \text { [43] }\end{array}$ & $\begin{array}{l}\text { Active Laser } \\
\text { Treatment }\end{array}$ & $\begin{array}{l}\text { Inactive Dummy } \\
\text { Treatment }\end{array}$ & Unclear & TSI & 4 Weeks & No & $\begin{array}{c}\text { No Statistically Significant } \\
\text { Improvement Neither in Laser nor In } \\
\text { Control Group }\end{array}$ & No \\
\hline $\begin{array}{c}\text { Doi, } \\
2016[51]\end{array}$ & Acupuncture & No Treatment & $\begin{array}{l}\text { Randomization } \\
\text { Was Carried Out } \\
\text { with The Aid of } \\
\text { Computerized } \\
\text { Table of Random } \\
\text { Numbers } \\
\text { Created by A } \\
\text { Microsoft Excel } \\
\text { Spreadsheet. }\end{array}$ & VAS, THI & 5 Weeks & No & $\begin{array}{l}\text { Treatment with Acupuncture } \\
\text { Improves the Perception of Tinnitus, } \\
\text { Decreases the Intensity Level, Hence } \\
\text { There Is No Comparison Between } \\
\text { Levels of Improvement }\end{array}$ & $\begin{array}{c}\text { Yes, } \\
\text { Against } \\
\text { Placebo In } \\
5 \text { Weeks, } \\
\text { However No } \\
\text { Comparison } \\
\text { of Decrease }\end{array}$ \\
\hline $\begin{array}{c}\text { Dos Santos, } \\
2014 \text { [38] }\end{array}$ & $\begin{array}{l}\text { Hearing Aids + } \\
\text { Sound Generator }\end{array}$ & Hearing Aids & Unclear & THI & 3 Months & $80,0 \%$ & $\begin{array}{l}\text { No Superiority of The Combined Use } \\
\text { of Amplification and Sound Generator } \\
\text { Over Conventional Amplification } \\
\text { Alone in Reducing the Discomfort of } \\
\text { Tinnitus. Both Groups Presented } \\
\text { Similar Responses in Both Reduction } \\
\text { of Discomfort Caused by Tinnitus }\end{array}$ & No \\
\hline $\begin{array}{l}\text { El Beaino, } \\
2018[77]\end{array}$ & Sulodexide & Placebo & Unclear & THI, Mini TQ & $\begin{array}{l}\text { Right After } \\
\text { Treatment }\end{array}$ & $80 \%$ & $\begin{array}{l}\text { Improvement in THI and Mini TQ } \\
\text { Right After the End of Treatment with } \\
\text { Sulodexide }\end{array}$ & Yes \\
\hline $\begin{array}{l}\text { Elzayat, } \\
2018 \text { [64] }\end{array}$ & $\begin{array}{c}\text { Group A Was } \\
\text { Injected with } \\
\text { Combined Lidocaine } \\
2 \% \text { And } \\
\text { Dexamethasone } \\
8 \mathrm{Mg} / 2 \mathrm{~mL} \text { (ITLD). } \\
\text { Group B Was Injected } \\
\text { Only by } \\
\text { Dexamethasone } \\
8 \mathrm{Mg} / 2 \mathrm{ML} \text {. (ITD). }\end{array}$ & $\begin{array}{c}\text { ITD As A } \\
\text { Controlled Group }\end{array}$ & Clear & THI, VAS, ATQ & 6 Months & No & $\begin{array}{c}\text { Both Treatments Were Effective but } \\
\text { No Difference Between Groups } \\
\text { Was Found }\end{array}$ & Yes \\
\hline
\end{tabular}


Table 2. Cont.

\begin{tabular}{|c|c|c|c|c|c|c|c|c|}
\hline $\begin{array}{l}\text { Author, } \\
\text { Year }\end{array}$ & Treatment & $\begin{array}{l}\text { Control Group } \\
\text { Intervention }\end{array}$ & Randomization & Outcome Measures & $\begin{array}{l}\text { Monitoring } \\
\text { Duration }\end{array}$ & $\begin{array}{l}\text { Power } \\
\text { Analysis }\end{array}$ & Results & $\begin{array}{l}\text { Is Treatment } \\
\text { Effective? }\end{array}$ \\
\hline $\begin{array}{l}\text { Folmer, } \\
2015[45]\end{array}$ & $\begin{array}{l}\text { rTMS Daily For } \\
2 \text { Weeks }\end{array}$ & $\begin{array}{l}\text { Sham Rtms With } \\
\text { A Same Looking } \\
\text { Coil }\end{array}$ & Unclear & TFI & 26 Months & No & $\begin{array}{l}\text { Significant Improvement in Active } \\
\text { Compared to Placebo Group }\end{array}$ & Yes \\
\hline $\begin{array}{l}\text { Godbehere, } \\
2019 \text { [79] }\end{array}$ & Theta Burst TMS & Placebo Arm & Unclear & TFI & 4 Weeks & No & $\begin{array}{l}\text { No Significant Difference in Scores } \\
\text { Between the Active Treatment Group } \\
\text { and The Sham Control Group }\end{array}$ & No \\
\hline $\begin{array}{c}\text { Han, } \\
2012 \text { [22] }\end{array}$ & Clonazepam & Ginkgo Biloba & Unclear & THI, VAS, Loudness Scale & & No & $\begin{array}{c}\text { Improvement with Use of } \\
\text { Clonazepam and Not Gingko Biloba, } \\
\text { but Right After Treatment }\end{array}$ & Yes \\
\hline $\begin{array}{c}\text { Henry, } \\
2016 \text { [52] }\end{array}$ & TM-TRT-TED & No Treatment & Clear & THI & 18 Months & $80 \%$ & $\begin{array}{c}\text { No Statistically Significant } \\
\text { Improvement In THI. By } 6 \text { Months, } \\
\text { The TED Group Showed Significant } \\
\text { Improvement from Baseline and Its } \\
\text { Improvement Was Not Significantly } \\
\text { Different from That Shown in TM } \\
\text { Or TRT. }\end{array}$ & No \\
\hline $\begin{array}{l}\text { Hesser, } \\
2012[23]\end{array}$ & CBT Or ACT & $\begin{array}{l}\text { Monitored } \\
\text { Internet } \\
\text { Discussion } \\
\text { Forum }\end{array}$ & Clear & $\begin{array}{c}\text { Primary: THI, Secondary: } \\
\text { HADS }\end{array}$ & 1 Year & $80 \%$ & $\begin{array}{l}\text { The Effect of ACT Compared with The } \\
\text { Control Condition at Posttreatment on } \\
\text { The Primary Outcome Was in The } \\
\text { Moderate Range and Comparable to } \\
\text { The Effect Observed Following CBT } \\
\text { (D = } 0.68 \text { vs. } \mathrm{D}=0.70) \text {. }\end{array}$ & No \\
\hline $\begin{array}{c}\text { Hoare, } \\
2012 \text { [24] }\end{array}$ & Frequency Training & $\begin{array}{l}\text { Different } \\
\text { Frequency } \\
\text { Training }\end{array}$ & & THQ & 4 Weeks & $80 \%$ & $\begin{array}{l}\text { Statistically and Clinically Meaningful } \\
\text { Improvement in All Groups. No } \\
\text { Difference Between Groups }\end{array}$ & Yes \\
\hline $\begin{array}{l}\text { Hoare, } \\
2014[39]\end{array}$ & $\begin{array}{l}\text { To Play A Tailored } \\
\text { Video Game For } 30 \\
\text { Minutes, } 5 \text { Days A } \\
\text { Week For } 4 \text { Weeks }\end{array}$ & $\begin{array}{l}\text { Another Type Of } \\
\text { FDT }\end{array}$ & Clear & THQ & 4 Weeks & $80 \%$ & $\begin{array}{c}\text { Statistically but Not Clinically } \\
\text { Significant Changes in One of The } \\
\text { Games Used }\end{array}$ & No \\
\hline
\end{tabular}


Table 2. Cont.

\begin{tabular}{|c|c|c|c|c|c|c|c|c|}
\hline $\begin{array}{l}\text { Author, } \\
\text { Year }\end{array}$ & Treatment & $\begin{array}{l}\text { Control Group } \\
\text { Intervention }\end{array}$ & Randomization & Outcome Measures & $\begin{array}{l}\text { Monitoring } \\
\text { Duration }\end{array}$ & $\begin{array}{c}\text { Power } \\
\text { Analysis }\end{array}$ & Results & $\begin{array}{l}\text { Is Treatment } \\
\text { Effective? }\end{array}$ \\
\hline $\begin{array}{l}\text { Hoekstra, } \\
2013[33]\end{array}$ & rTMS in $1000 \mathrm{~Hz}$ & Placebo & Unclear & $\begin{array}{c}\text { Primary: TQ. Secondary } \\
\text { THI, VAS }\end{array}$ & 6 Months & $80 \%$ & $\begin{array}{l}\text { No Significant Difference } \\
\text { Between Groups }\end{array}$ & No \\
\hline $\begin{array}{l}\text { Hong, } \\
2018[78]\end{array}$ & $\begin{array}{l}40 \text { Minutes Session of } \\
\text { Nitrous Oxide Under } \\
\text { General Anesthesia }\end{array}$ & $\begin{array}{l}\text { Same Procedure } \\
\text { Without Nitrous } \\
\text { Oxide }\end{array}$ & Clear & TFI & 2 Weeks & $81 \%$ & $\begin{array}{l}\text { No Significant Differences Between } \\
\text { Intervention and Control Group. } \\
\text { Neither Groups Had Clinical or } \\
\text { Statistically Significant Improvement }\end{array}$ & No \\
\hline $\begin{array}{c}\text { Jeon, } \\
2012[25]\end{array}$ & Acupuncture & Sham & Unclear & THI, VAS & & No & $\begin{array}{l}\text { No Significant Differences Between } \\
\text { Acupuncture and Sham }\end{array}$ & No \\
\hline $\begin{array}{l}\text { Kallogjeri, } \\
2017[65]\end{array}$ & $\begin{array}{l}\text { Brain fitness program } \\
\text { tinnitus (BFP-T) }\end{array}$ & No Treatment & Unclear & $\begin{array}{l}\text { THI, TFI, Global Bother } \\
\text { Score }\end{array}$ & 8 Weeks & $85 \%$ & $\begin{array}{l}\text { No Statistically Significant Changes } \\
\text { Between Study Groups. }\end{array}$ & No \\
\hline $\begin{array}{c}\text { Kim, } \\
2017[66]\end{array}$ & Manual Acupuncture & Electroacupuncture & Unclear & THI, VAS & & $80 \%$ & $\begin{array}{l}\text { No Significant Improvement for Any } \\
\text { Acupuncture Group In Regards To } \\
\text { THI and Loudness }\end{array}$ & No \\
\hline $\begin{array}{l}\text { Kreuzer, } \\
2012[26]\end{array}$ & $\begin{array}{l}\text { Mindfulness and } \\
\text { Body Group Therapy }\end{array}$ & $\begin{array}{l}\text { Waiting List } \\
\text { (Therapy After } \\
24 \text { Weeks) }\end{array}$ & Unclear & TQ & 24 Weeks & No & $\begin{array}{c}\text { A Significant Reduction in The TQ } \\
\text { Score (Baseline vs. Week 9) Compared } \\
\text { to The Waiting List Control Group, } \\
\text { However Difference Was Not Stable } \\
\text { in Long Term F/U }\end{array}$ & No \\
\hline $\begin{array}{l}\text { Kreuzer, } \\
2015 \text { [46] }\end{array}$ & $\begin{array}{l}\text { Medial Frontal } \\
\text { Stimulation with } \\
\text { Double Cone Coil + } \\
\text { Stimulation of The } \\
\text { Left } \\
\text { Temporo-Parietal } \\
\text { Junction Area }\end{array}$ & $\begin{array}{c}\text { Conventional } \\
\text { Prefrontal Left } \\
\text { DLPFC- } \\
\text { Stimulation + } \\
\text { Stimulation of } \\
\text { The Left } \\
\text { Temporo-Parietal } \\
\text { Junction Area }\end{array}$ & Unclear & $\begin{array}{c}\text { TQ, Secondary: THI, } \\
\text { CGI-CHANGE, } \\
\text { Whoqol-Bref-Questionnaire }\end{array}$ & 12 Weeks & No & $\begin{array}{l}\text { TICDC-Stimulation Non-Superior to } \\
\text { Standard Rtms Regarding Both } \\
\text { Primary and Secondary } \\
\text { Outcome Measures. }\end{array}$ & No \\
\hline
\end{tabular}


Table 2. Cont.

\begin{tabular}{|c|c|c|c|c|c|c|c|c|}
\hline $\begin{array}{l}\text { Author, } \\
\text { Year }\end{array}$ & Treatment & $\begin{array}{l}\text { Control Group } \\
\text { Intervention }\end{array}$ & Randomization & Outcome Measures & $\begin{array}{l}\text { Monitoring } \\
\text { Duration }\end{array}$ & $\begin{array}{l}\text { Power } \\
\text { Analysis }\end{array}$ & Results & $\begin{array}{l}\text { Is Treatment } \\
\text { Effective? }\end{array}$ \\
\hline $\begin{array}{l}\text { Landgrebe, } \\
2017 \text { [67] }\end{array}$ & $\begin{array}{l}2 \text { Week Treatment } \\
\text { Real 1-Hz-Rtms vs. } \\
\text { Sham Rtms }\end{array}$ & Sham Rtms & Clear & $\begin{array}{l}\text { Primary: The Change of } \\
\text { Tinnitus Severity Assessed } \\
\text { by Means of The Change of } \\
\text { The TQ Sum Score Between } \\
\text { Baseline Score vs. Day } 12 . \\
\text { Secondary: Changes of The } \\
\text { TQ Sum Score, The THI and } \\
\text { TSS During the Treatment } \\
\text { and The Follow-Up Period. } \\
\text { Further: Changes of Overall } \\
\text { Illness Severity, Changes in } \\
\text { Depressive Symptoms, } \\
\text { Changes in Quality of Life } \\
\text { and Changes in } \\
\text { Psychoacoustic Measures of } \\
\text { Tinnitus. }\end{array}$ & 26 Weeks & No & $\begin{array}{l}\text { Real 1-Hz-Rtms Applied to The Left } \\
\text { Temporal Cortex Did Not Provide } \\
\text { Any Therapeutic Benefit as Compared } \\
\text { to Sham Treatment in Patients with } \\
\text { Chronic Tinnitus. }\end{array}$ & No \\
\hline $\begin{array}{l}\text { Laureano, } \\
2016 \text { [53] }\end{array}$ & $\begin{array}{c}\text { True } \\
\text { Acupuncture } \\
\text { 99mTC-ECD SPECT }\end{array}$ & $\begin{array}{c}\text { Sham } \\
\text { Acupuncture }\end{array}$ & Unclear & $\begin{array}{c}\text { Primary: SPECT } \\
\text { Measurements, Secondary: } \\
\text { THI, VAS, HAS, BDI }\end{array}$ & 12 Weeks & $80 \%$ & $\begin{array}{l}\text { No Significant Differences After } \\
\text { Treatment Were Observed with } \\
\text { Regard to the VAS, HAS or BDI } \\
\text { Between the Treatment Groups. }\end{array}$ & No \\
\hline $\begin{array}{c}\text { Lee, } \\
2018[74]\end{array}$ & IT Steroids & Placebo (Saline) & Clear & THI, VAS & 1 Month & $80 \%$ & $\begin{array}{l}\text { No Difference Between IT And } \\
\text { Placebo Groups }\end{array}$ & No \\
\hline $\begin{array}{l}\text { Lehner, } \\
2016 \text { [54] }\end{array}$ & $\begin{array}{l}\text { High Frequency } \\
\text { rTMS }\end{array}$ & Single Site Rtms & Clear & TQ, THI, & 6 Months & $80 \%$ & No Difference Between Groups & No \\
\hline $\begin{array}{c}\mathrm{Li}, \\
2016 \text { [55] }\end{array}$ & $\begin{array}{l}\text { Music Altered by The } \\
\text { Software to Treat } \\
\text { Tinnitus }\end{array}$ & Unaltered Music & Unclear & THI, TFI, HADS & 12 Months & $80 \%$ & $\begin{array}{l}\text { Statistically Significant and Clinically } \\
\text { Meaningful Effects of The Therapy as } \\
\text { Indicated by The Consistent } \\
\text { Treatment-Control Group Difference } \\
\text { in THI Score and The Significant } \\
\text { Reduction in THI Score Within the } \\
\text { Treatment Group During The } \\
\text { 12-Month Period. }\end{array}$ & Yes \\
\hline
\end{tabular}


Table 2. Cont.

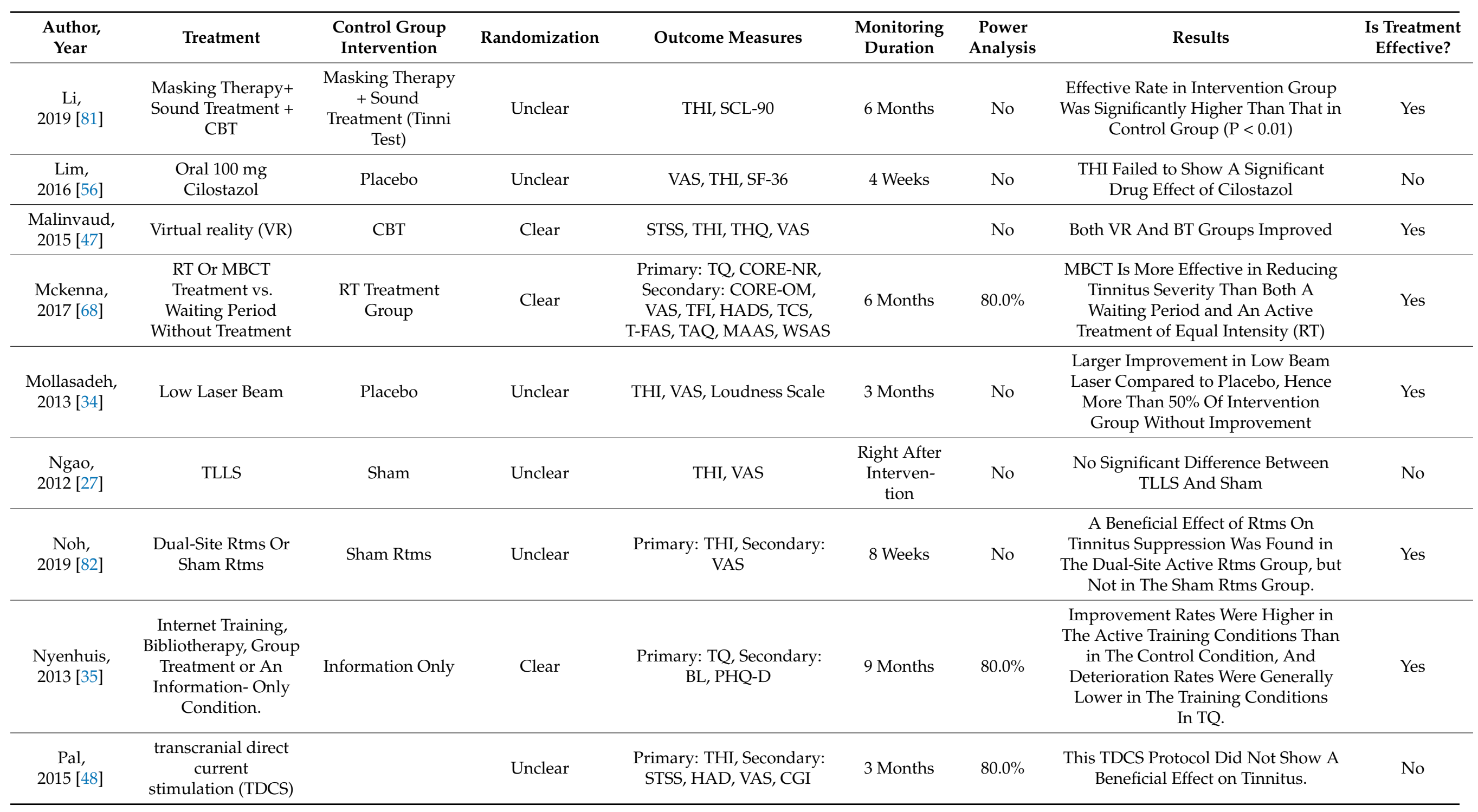


Table 2. Cont.

\begin{tabular}{|c|c|c|c|c|c|c|c|c|}
\hline $\begin{array}{l}\text { Author, } \\
\text { Year }\end{array}$ & Treatment & $\begin{array}{l}\text { Control Group } \\
\text { Intervention }\end{array}$ & Randomization & Outcome Measures & $\begin{array}{l}\text { Monitoring } \\
\text { Duration }\end{array}$ & $\begin{array}{l}\text { Power } \\
\text { Analysis }\end{array}$ & Results & $\begin{array}{c}\text { Is Treatment } \\
\text { Effective? }\end{array}$ \\
\hline $\begin{array}{l}\text { Plewnia, } \\
2012[28]\end{array}$ & $\begin{array}{l}\text { CTBS Over the } \\
\text { Secondary Auditory } \\
\text { Cortex (SAC), The } \\
\text { Temporoparietal } \\
\text { Association Cortex } \\
\text { (TAC), Or Sham } \\
\text { Stimulation } \\
\text { [Placebo (PLC)]. }\end{array}$ & Placebo & Clear & TQ & 3 Months & $80 \%$ & $\begin{array}{l}\text { No Difference Between Real and } \\
\text { Sham Treatments nor Between } \\
\text { Temporal and Temporoparietal Ctbs. }\end{array}$ & No \\
\hline $\begin{array}{l}\text { Prozchazkova, } \\
2019 \text { [83] }\end{array}$ & Ginkgo Biloba & Pentoxifylline & Unclear & VAS, Mini TQ, HADS & 3 Months & No & $\begin{array}{l}\text { Both Gingko Biloba and } \\
\text { Pentoxifylline Improve Mini TQ. No } \\
\text { Difference Between Groups }\end{array}$ & Yes \\
\hline $\begin{array}{l}\text { Radunz, } \\
2019 \text { [84] }\end{array}$ & Gingko Biloba & HA & Unclear & THI, VAS & 6 Months & No & $\begin{array}{l}\text { Both Gingko Biloba Improved } \\
\text { Compared to Baseline, No Difference } \\
\text { Between Groups Though, Apart from } \\
\text { Long Lasting Tinnitus }\end{array}$ & Yes \\
\hline $\begin{array}{l}\text { Rocha, } \\
2012 \text { [29] }\end{array}$ & $\begin{array}{l}10 \text { Sessions of } \\
\text { Myofascial Trigger } \\
\text { Point Deactivation }\end{array}$ & $\begin{array}{l}10 \text { Sessions with } \\
\text { Sham } \\
\text { Deactivation }\end{array}$ & Unclear & THI & 3 Months & No & $\begin{array}{l}\text { MTP Deactivation Through Digital } \\
\text { Pressure Was Deemed Effective in } \\
\text { Each and Every Tinnitus Variable } \\
\text { Under Evaluation and In the Medium } \\
\text { Run Responsiveness to Treatment } \\
\text { Remained Stable In } 75.8 \% \text { Patients. }\end{array}$ & Yes \\
\hline $\begin{array}{c}\text { Rojas- } \\
\text { Roncancio, } \\
2016 \text { [57] }\end{array}$ & $\begin{array}{l}\text { Manganese and } \\
\text { Lipoflavonoid Plus }\end{array}$ & $\begin{array}{l}\text { Lipoflavonoid } \\
\text { Plus }\end{array}$ & Unclear & THQ, TPFQ & 6 Months & No & $\begin{array}{l}\text { No Significant Improvement in } \\
\text { Both Groups }\end{array}$ & No \\
\hline $\begin{array}{l}\text { Roland, } \\
2016[58]\end{array}$ & $\begin{array}{l}\text { Sham or Active } \\
\text { Treatment rTMS } \\
\text { to TPJ }\end{array}$ & Sham Rtms to TPJ & Clear & & 2 or 4 Weeks & No & $\begin{array}{l}\text { No Changes in Neural Connectivity } \\
\text { Following Rtms Therapy. Results } \\
\text { Suggest Instead That the TPJ May Not } \\
\text { Be an Ideal Target for Tinnitus } \\
\text { Treatment. }\end{array}$ & No \\
\hline $\begin{array}{l}\text { Sahlsten, } \\
2017[71]\end{array}$ & rTMS & Placebo Rtms & Unclear & THI, VAS & 6 Months & $80 \%$ & $\begin{array}{l}\text { Improvement for The VAS Scores } \\
\text { (Intensity, Annoyance, Distress) And } \\
\text { THI Scores Both in The Active Rtms } \\
\text { Group and The Placebo Group. }\end{array}$ & No \\
\hline
\end{tabular}


Table 2. Cont.

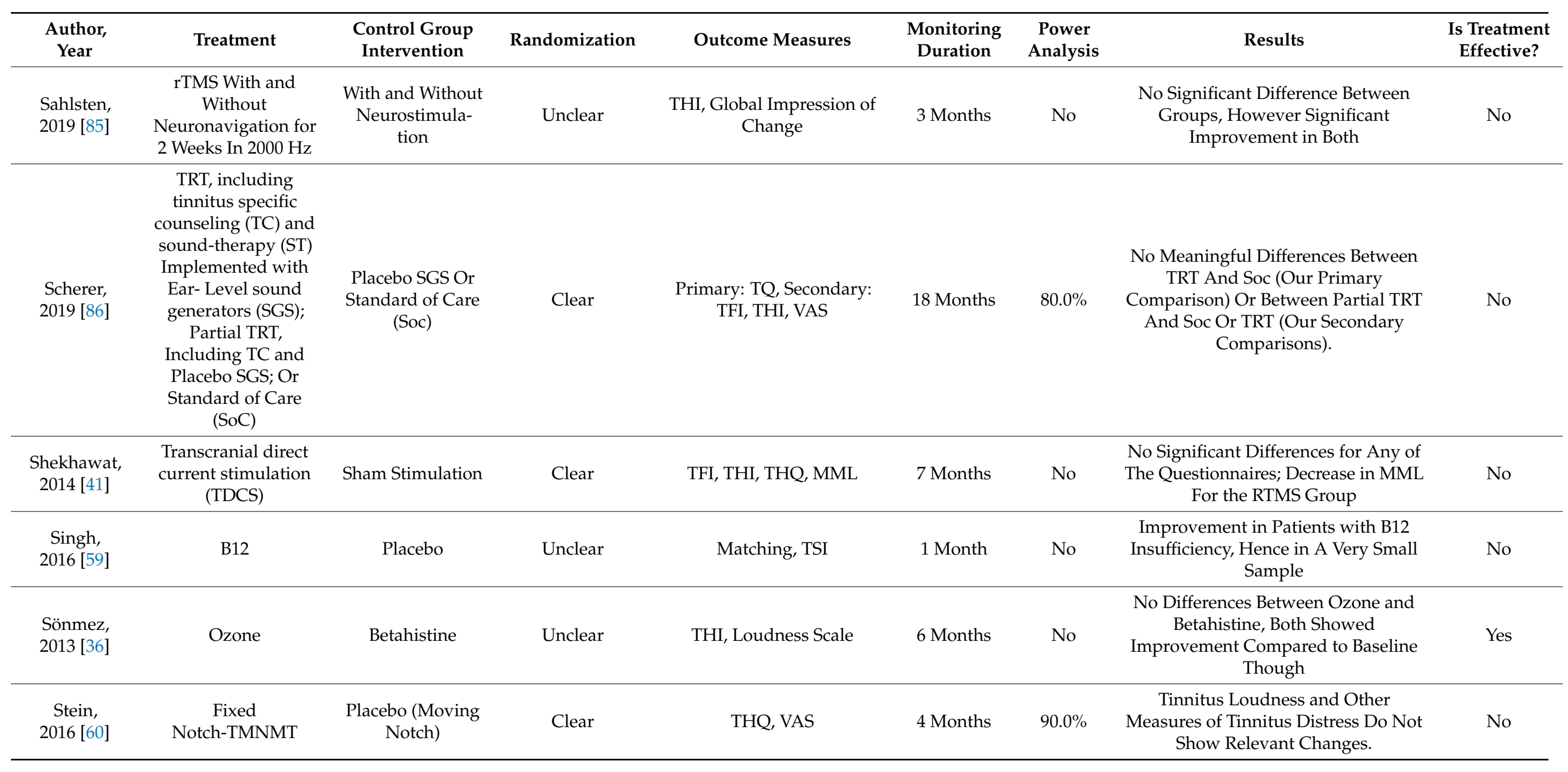


Table 2. Cont.

\begin{tabular}{|c|c|c|c|c|c|c|c|c|}
\hline $\begin{array}{l}\text { Author, } \\
\text { Year }\end{array}$ & Treatment & $\begin{array}{l}\text { Control Group } \\
\text { Intervention }\end{array}$ & Randomization & Outcome Measures & $\begin{array}{l}\text { Monitoring } \\
\text { Duration }\end{array}$ & $\begin{array}{l}\text { Power } \\
\text { Analysis }\end{array}$ & Results & $\begin{array}{l}\text { Is Treatment } \\
\text { Effective? }\end{array}$ \\
\hline $\begin{array}{l}\text { Sziklai, } \\
2011[19]\end{array}$ & Pramipexole & Placebo & Clear & THI & 4 Weeks & No & $\begin{array}{l}\text { No Cumulative Analysis. Greater } \\
\text { Proportion of Patients Reporting } \\
\text { Tinnitus Disappearance in The } \\
\text { Interventional Group. Results Not } \\
\text { Confirmed by Electrocochleography. }\end{array}$ & Unclear \\
\hline $\begin{array}{l}\text { Taslimi, } \\
2013 \text { [37] }\end{array}$ & Ondansedron & Placebo & Clear & THI, TSI, VAS, HADS & & No & $\begin{array}{c}\text { No Significant Differences Between } \\
\text { Ondansedron And Placebo }\end{array}$ & No \\
\hline $\begin{array}{c}\text { Tass, } \\
2012[30]\end{array}$ & CR & Placebo & & VAS, TQ & 12 Weeks & No & $\begin{array}{c}\text { Improvement Before and After } \\
\text { Treatment and Also Compared to } \\
\text { Placebo }\end{array}$ & Yes \\
\hline $\begin{array}{l}\text { Teismann, } \\
2014[42]\end{array}$ & $\begin{array}{l}\text { Anodal TDCS, } \\
\text { Cathodal TDCS + } \\
\text { TMNMT }\end{array}$ & $\begin{array}{l}\text { Sham Stimulation } \\
+ \text { TMNMT }\end{array}$ & Unclear & THQ, THI, TQ & 31 Days & No & $\begin{array}{c}\text { No Significant Modulating Effect of } \\
\text { TDCS Polarity: Significant Main } \\
\text { Effects or Interactions of TDCS } \\
\text { Condition Were Neither Found in The } \\
\text { Primary Outcome Measure nor In } \\
\text { Any of The Secondary Outcome } \\
\text { Measures (THI, TQ, Or Loudness } \\
\text { VAS; }\end{array}$ & No \\
\hline $\begin{array}{l}\text { Thabit, } \\
2015 \text { [49] }\end{array}$ & rTMS & RTMS & Unclear & THI, VAS & 1 Month & No & $\begin{array}{c}\text { Combination Treatment Significantly } \\
\text { Better }\end{array}$ & Yes \\
\hline $\begin{array}{l}\text { Theodoroff, } \\
2017 \text { [72] }\end{array}$ & $\begin{array}{l}\text { LEVO System with A } \\
\text { Tinnitus-Matched } \\
\text { Stimulus (TM Group) } \\
\text { vs. LEVO System } \\
\text { with A Noise } \\
\text { Stimulus (NS Group; } \\
\text { White Noise And/or } \\
\text { Band Noise) vs. } \\
\text { Marsona } 1288 \text { Sound } \\
\text { Condi- } \\
\text { tioner/Tinnitus } \\
\text { Masker (Bedside } \\
\text { Sound Generator } \\
\text { Device; BSG Group). }\end{array}$ & $\begin{array}{l}\text { BSG And NS } \\
\text { Groups, but Not } \\
\text { in The Same } \\
\text { Manner as A } \\
\text { Placebo- } \\
\text { Controlled } \\
\text { Group }\end{array}$ & Unclear & TFI, NRS, And LM at $1 \mathrm{kHz}$ & 3 Months & No & $\begin{array}{l}\text { Greater Average Improvement in } \\
\text { Reactions to Tinnitus with TM or NS } \\
\text { Devices Compared to The } \\
\text { BSG Device. }\end{array}$ & Yes \\
\hline
\end{tabular}


Table 2. Cont.

\begin{tabular}{|c|c|c|c|c|c|c|c|c|}
\hline $\begin{array}{l}\text { Author, } \\
\text { Year }\end{array}$ & Treatment & $\begin{array}{l}\text { Control Group } \\
\text { Intervention }\end{array}$ & Randomization & Outcome Measures & $\begin{array}{l}\text { Monitoring } \\
\text { Duration }\end{array}$ & $\begin{array}{c}\text { Power } \\
\text { Analysis }\end{array}$ & Results & $\begin{array}{l}\text { Is Treatment } \\
\text { Effective? }\end{array}$ \\
\hline $\begin{array}{c}\text { Tutar, } \\
2020[88]\end{array}$ & $\begin{array}{l}10 \text { Sessions Of } 30 \\
\text { Minutes in One } \\
\text { Month }\end{array}$ & Placebo & Unclear & THI, DASS & 4 Weeks & No & $\begin{array}{l}\text { Significant Improvement in Uni- And } \\
\text { Bilateral Groups Compared to Placebo }\end{array}$ & Yes \\
\hline $\begin{array}{c}\text { Tyler, } \\
2017[73]\end{array}$ & VNS Implant-Paired & $\begin{array}{l}\text { VNS Implant- } \\
\text { Unpaired (Paired } \\
\text { After } 6 \text { Weeks) }\end{array}$ & Clear & THI, TFI, THQ & 1 Year & No & $\begin{array}{l}\text { No Significant Differences for Any of } \\
\text { The Outcome Measures }\end{array}$ & No \\
\hline $\begin{array}{l}\text { Westin, } \\
2011[20]\end{array}$ & ACT, TRT & Wait List Control & Unclear & $\begin{array}{l}\text { Primary: THI, Secondary: } \\
\text { ISI, QOLI, HADS, CGI-I }\end{array}$ & 18 Months & $80 \%$ & $\begin{array}{c}\text { ACT Is More Effective in Reducing } \\
\text { Tinnitus Impact Than Tinnitus } \\
\text { Retraining Therapy or Being on A } \\
\text { Wait List. }\end{array}$ & Yes \\
\hline $\begin{array}{c}\text { Wise, } \\
2016[62]\end{array}$ & $\begin{array}{l}\text { Experimental } \\
\text { Attention Training } \\
\text { Game (“Terrain") }\end{array}$ & $\begin{array}{l}\text { A Control Game } \\
\text { ("Tetris") }\end{array}$ & Unclear & $\begin{array}{c}\text { TFI, Secondary: THI, } \\
\text { Tinnitus Severity Numeric } \\
\text { Scales }\end{array}$ & 20 Days & $92 \%$ & $\begin{array}{l}\text { TFI Scores Improved Following The } \\
\text { 20-Day Use for the "Terrain" Program } \\
\text { Compared with The Nonauditory } \\
\text { "Tetris" Group. }\end{array}$ & Yes \\
\hline $\begin{array}{l}\text { Yakunina, } \\
2019[87]\end{array}$ & $\begin{array}{l}\text { HAs With WDRC, } \\
\text { HAs With FT, Or } \\
\text { HAs With LFT }\end{array}$ & $\begin{array}{l}\text { FL Techniques } \\
\text { (LFT and FT) } \\
\quad \text { Group }\end{array}$ & Clear & $\begin{array}{c}\text { Primary: THI, Secondary: } \\
\text { VAS }\end{array}$ & 6 Months & $80.0 \%$ & $\begin{array}{l}\text { No Significant Differences Were } \\
\text { Found Between Conventional Has } \\
\text { And FL-Type Has in Terms of Tinnitus } \\
\text { Relief Among Patients With HFHL. }\end{array}$ & No \\
\hline $\begin{array}{l}\text { Zarenoe, } \\
2016[63]\end{array}$ & $\begin{array}{l}\text { MI Group Received } \\
\text { A Brief MI Program, } \\
\text { Whereas Patients in } \\
\text { The SP Group } \\
\text { Underwent } \\
\text { Conventional } \\
\text { Hearing Aid Fitting. }\end{array}$ & $\begin{array}{l}\text { Conventional } \\
\text { Hearing Aid } \\
\text { Fitting Group }\end{array}$ & Unclear & THI, IOI-HA & 3 Months & No & $\begin{array}{l}\text { The MI Intervention Did Not Appear } \\
\text { to Have Any Additional Effect on } \\
\text { Hearing Aid Fitting Compared to } \\
\text { Conventional Hearing Rehabilitation. }\end{array}$ & No \\
\hline
\end{tabular}




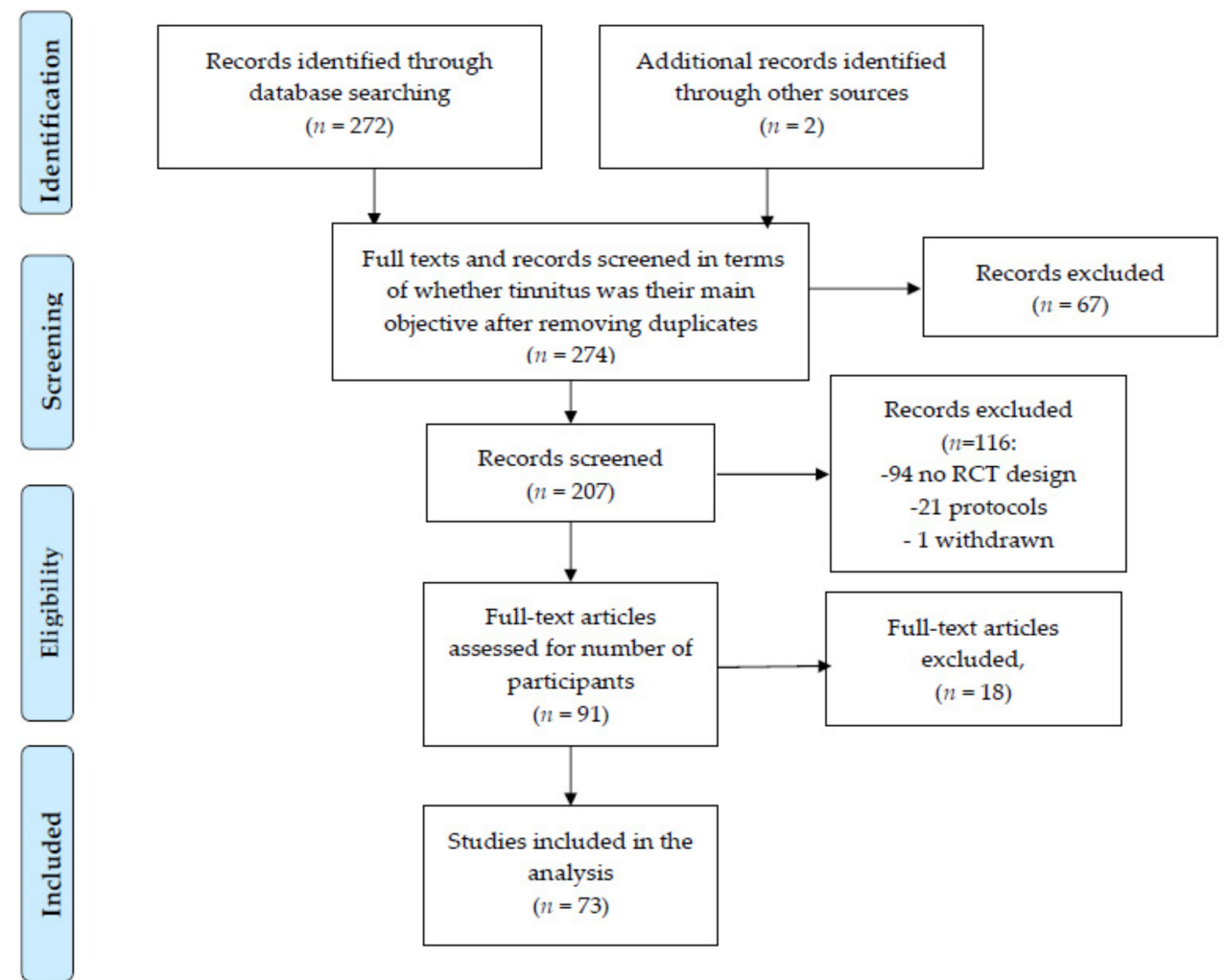

Figure 1. Study selection PRISMA flow diagram.

\subsubsection{Methods and Study Procedures}

Median number of participants per RCT was as low as 54, whereas 11 of the included studies had a sample size of more than 100 subjects. The vast majority of the studies (46 out of 73) did not provide power analysis nor details on the sample size calculation prior to the study execution.

Across studies, randomization procedure was not described in a clear manner in 46 of the studies $(62.16 \%)$ (absence of specific methodology, inadequacy of the reported method to the study design).

Primary objective of the included RCTs is the evaluation of specific therapeutic approaches; seventeen of them focus on transcranial magnetic stimulation (TMS) [28,33,41,42, $44,45,48,54,67,71,72,76,79,82,85,89-91]$, four on acupuncture as monotherapy $[25,51,53,66]$, more than ten on cognitive behavioral treatment, relaxation and mindfulness $[20,21,23$, $26,35,40,47,61,65,68-70,75,81,92]$, seven on provision of hearing aids $[38,41,63,84,87,93,94]$, 8 on sound therapy $[24,39,55,60,86,95-97]$, two on electrical stimulation $[29,73,88]$, while more than twenty evaluate the efficacy of various pharmacological interventions [18,19, $22,31,36,37,50,56-59,64,74,77,78,80,83,84,93]$ and one the efficacy of the Acoustic CR neuromodulation device [30].

The duration of participants' follow-up ranged from immediate after treatment up to 26 months $[18,98]$.

\subsubsection{Methods and Outcome Measures}

Outcome measures used across studies vary and range from simplistic VAS to specialized and cross-culturally validated questionnaires; 14 studies have used the TFI; 9 the THI; 9 the THQ; 2 the TAQ. The majority of studies use more than one questionnaire, covering tinnitus and its comorbidities (e.g., the Hospital Anxiety and Depression Scale or HADS and the Patient Health Questionnaire-Depression or PHQ-D) (for more details, see Table 2). 


\section{Discussion}

\subsection{Tinnitus Duration and Intermittent Character}

Tinnitus is a subjective symptom and in many cases it fluctuates over time [1]. Typically, patients report either fluctuations that might or might not be influenced by external factors or by their emotional status, for example levels of environmental noise or stress [1]. In the vast majority of studies, the outcome measures consist of questionnaires that are handed out at specific time points and supposed to evaluate a certain period of time [6]. This method has a fundamental flaw by default: even if patients are asked to provide information about the tinnitus severity over a defined time period (e.g., one week), the tinnitus severity at the moment when the questionnaire is filled out dominates. Tinnitus fluctuations over time or even periods without tinnitus are typically not sufficiently reflected due to memory and reporting bias. When patients are asked to fill in the questionnaire, the results will depend on their emotional status in general and particularly at the time information was provided, their overall attitude and tinnitus perception, and also on their tendency to focus on negative aspects. These confounding factors influence both the presence as well as the level of annoyance and consequently the tinnitus reporting. Therefore, they can be considered as an intrinsic difficulty that is present as a systematic bias across tinnitus related studies.

A potential solution to this could be the use of ecological momentary assessment, which is commonly integrated through mobile applications and allows ongoing recording of fluctuations in tinnitus severity as well as the correlation with certain incidents and behaviors which are captured at the same time (e.g., environmental noise, road traffic, etc.) [12]. This approach, if not well designed or capable of adjustments, may contradict efforts of habituation, since it requires that patients be frequently occupied with their tinnitus and its characteristics.

Only one of the studies took into account the duration of tinnitus within specific time intervals [80]. This finding is remarkable, as an expert consensus initiative from 2007 for tinnitus assessment and outcome measurement proposed that tinnitus patients should be asked about which percentage of their time they perceive their tinnitus?" [99].

Of course, even if asked, this information would be difficult to collect, due to recall bias and inability of patients to provide reliable information in regards to tinnitus duration due to different factors commonly mentioned, including fluctuation of tinnitus occurrence and perceived loudness if present, masking in noisy environments and lack of focus. Intrinsic issues already mentioned have not allowed a universally accepted answer to fundamental question in regards to tinnitus fluctuations, like whether patients with intermittent tinnitus tend to experience less annoyance or whether their treatment response is expected to be better [100]. Consequently, tinnitus duration and fluctuation and whether it was intermittent or not and under which conditions, was not taken into account as a factor in data analysis and interpretation neither, which is a possibly interesting point that should be considered by future studies.

\subsection{Level of Perceived Annoyance}

One of the few things that are considered common ground in tinnitus literature is the fact that the majority of people with tinnitus do not consider their tinnitus bothersome [4]. Overall prevalence, often replicated in the introductory parts of tinnitus studies considered as exceeding $10 \%$ in the general population is based on surveys in large samples [4]. On one hand, in the generation of these epidemiological data, some methodological considerations might arise about the criterion used to define tinnitus. On the other hand, the phrasing of the probably largest survey ("In the past 12 months, have you been bothered by ringing, roaring, or buzzing in your ears or head that lasts for $5 \mathrm{~min}$ or more?) seems clear enough in terms of adequate duration (excluding brief spikes), type of sound (noise rather than hallucinations), and time frame (one year and not whole life time). In any case, reproducibility of similar numbers in different countries confirms that these estimations should be close to reality [101]. 
As expected, the number of tinnitus sufferers seeking help by health professionals are much lower than the estimated prevalence [1]. This is of course easily explained by the fact that tinnitus is considered either as not a problem, or a small problem, often reported as non-bothersome. This also reflects to common clinical experience, according to which, a considerable group of patients with other chief complaints might mention tinnitus only when specifically asked. At the same time there is a subgroup of people with catastrophic tinnitus, who describe their tinnitus and the consequences as dramatical.

The discrepancy between the number of people with tinnitus and the number of those who seek medical help might also partly be due to the limited therapeutic options. A person suffering from tinnitus, who is told by the physician that there are no established possibilities to reduce the loudness of the tinnitus, might try to accept the situation without seeking further medical help.

As a result, it would be expected that tinnitus treatment studies, aiming to offer a solution to tinnitus sufferers, should select their participants accordingly and only include patients with a satisfactory level of annoyance, in order to fulfill a fundamental principle of medical research: ability to replicate their results to the target population.

According to the findings of our review, only 22 out of 73 RCTs clearly mention a minimum level of tinnitus annoyance in their inclusion criteria (Table 1). All popular questionnaires (THI, TFI, and TQ) are used, as well as Visual Analog Scales. THI is used in 12 of the studies, thresholds for inclusion however vary from 18 to 38 with four additional intermittent values: 20,25, and 30. Only one study has set both lower and upper limits, using TFI, in order to define a certain range [80].

Of course, it is reasonable to assume that individuals with non-bothersome tinnitus will not easily reach a tinnitus clinic and on top of this, be motivated for a usually demanding participation in an RCT. In addition, baseline values give an estimation of the overall annoyance.

Including a reasonable level of annoyance, using the outcome measures chosen for the specific RCT ideally not only setting lower but also upper limits, should be considered good practice in future RCTs.

\subsection{Tinnitus Audiological Characteristics}

Tinnitus frequency can vary from constant to less than weekly. There is also a considerable proportion of patients who state that their tinnitus is only detectable in the absence of any acoustic stimulation, typically before they fall asleep. On top of this, there is a wide range of sounds considered similar to the type of tinnitus sound. Typically, tinnitus pitch is better matched with high frequencies, although there is a considerable proportion of patients who either cannot easily identify a matching sound or better attribute to low frequencies [101]. Determination of tinnitus pitch, loudness, and minimum masking level can be useful in clinical practice, in spite of their questionable role and their fluctuating nature.

However, a robust relationship between tinnitus pitch, loudness and masking level and tinnitus prognosis and severity in terms of annoyance, functionality and handicap has not been established [102]. This means that in the studies investigating the effect of various treatments these characteristics are not useful as outcome parameter. This has been confirmed in our review, in which none of the studies used this type of data neither for outcome measurement, nor as a predictor for treatment outcome.

\subsection{Tinnitus and Hearing Loss}

It is widely reproduced in the literature that hearing loss is present in approximately $90 \%$ of tinnitus patients [4]. However, it is also common ground that existence as well as degree of hearing loss are not able to predict tinnitus occurrence and severity [1]. Since pathophysiology of tinnitus is complex and involves both auditory and brain function, it is impressive that hearing loss, although present in the vast majority of tinnitus patients, has not been thoroughly studied as a prognostic factor of tinnitus course, prognosis, and treatment response [1]. 
This gap is clearly reflected in the extracted literature. Only seven out of 73 studies clearly state in their inclusion criteria that tinnitus was considered as primary complaint by the participants (Table 1). It could be assumed that patients with hearing loss as their primary complaint would not be motivated to participate in tinnitus oriented RCTs. This means that an unknown proportion of study participants could have tinnitus, but not as primary complaint. The primary complaint could be hearing loss and tinnitus only the secondary complaint. Moreover, it is commonly seen in clinical practice that patients present with their primary complaint of tinnitus, but when they are clinically evaluated it is discovered that their main complaint and everyday handicap is their hearing loss. Consequently, and in accordance with common clinical experience, it could be hypothesized that these groups of patients are not homogenous in principle and combine patients in a wide spectrum between hearing loss and tinnitus as primary complaints-and all the shades in between. It is assumed that this could influence results and treatment response, especially in treatments like hearing aids. On top of this, even if identifying tinnitus as primary (but not only) complaint could potentially improve sample homogeneity, it could still exclude a significant group of patients who would consider hearing loss as their cardinal problem and also have adequately bothersome or even catastrophic tinnitus at the same time.

If just dealing with the existence of hearing loss is complex, taking the degree of hearing loss into account is even more challenging. More than one third of RCTs (25 out of 73) include a range of hearing loss in their inclusion criteria, whereas none of them analyzed the audiogram as a predictor for treatment response. Even those studies in which hearing levels were mentioned as inclusion criterion, they have typically vague descriptions of hearing functions, such as "normal hearing levels", hearing levels allowing conversation or mild, moderate, or severe hearing loss without an explicit definition or the respective thresholds.

In accordance, among the studies that evaluated other interventions than hearing aids, there was no study that took into consideration the use of hearing aids neither as an inclusion/exclusion criterion nor as a predictor nor as a confounder. The latter is potentially a hidden but significant risk of bias, since tinnitus improvement is considered as high as $55 \%$ in several case series analyzing hearing aids [5]. This could influence results in two ways; first, a selection bias, since only patients were included in the trial, in which hearing aid use was not effective to sufficiently decrease tinnitus annoyance; second, an unclear effect of a prolonged or recent use of hearing aids, which might influence the performance of an unknown proportion of hearing aids users both in the interventional and control arms. A clear exclusion of patients with a relatively recent hearing aid fitting should be considered as good practice in future RCTs.

The currently starting UNITI trial is strategically planned as an attempt to overcome the mentioned issues. Only patients with tinnitus as primary complaint will participate, and degree of hearing loss will be analyzed with sophisticated techniques as a potential confounder for treatment response. In addition, the efficacy of hearing aids as a sole measure to improve tinnitus will be tested for the first time in the context of a RCT against interventions based on other disciplines, like CBT [12].

\subsection{Remarks on Study Methodology}

Tinnitus interventions, in accordance with tinnitus pathophysiology, are heterogenous. In the RCTs collected, a wide spectrum of therapeutic strategies is performed ranging from transcranial magnetic and vagus nerve stimulation to internet-based CBT and altered/notched music (Table 2). A pattern that causes deviation from an optimal study design and is valid for various interventions is the inability to blind patients with respect to control interventions. For example, a blinded RCT comparing true and sham hearing aids is not feasible, since participants in the sham group will immediately recognize the sham devices, given that they will be unable to provide acoustic amplification. 
Apart from intrinsic limitations and barriers, tinnitus literature also suffers from methodological insufficiencies which are common in other fields, as well. Median number of participants per RCT is as low as 54, whereas only 10 exceed 100 participants. Most of the studies (46 out of 73) did not provide power analysis; hence, the rest reported power over $80 \%$. The same proportion of papers did not provide a clear, detailed, and reproducible description of their randomization procedure, a fact that clearly questions their qua-lity. Moreover, randomization procedure was found to be unclear in 46 of the studies (63.01\%) under the sense, that relevant information provided was generic and not adequate for the procedure to be replicated.

Although out of the scope of this review, what needs to be underlined is that many of the RCTs concerning tinnitus are supported by pharmaceutical companies or hearing and tinnitus-related devices manufacturers. So, results must always be read with caution and extra consideration for potential biases or conflicts of interests.

\subsection{Outcome Measures}

Tinnitus is a condition affecting everyday life in many ways, causing a list of issues including, but not limited to annoyance, functional disturbances, tinnitus intrusiveness and acceptance, disability for certain actions and tasks like concentration, ability to ignore negative emotions, sense of control, malaise, and loathsomeness. Hall et al. (2019) have recently suggested proper outcome measures for each type of intervention. These recommendations could not have been applied to the body of the RCTs examined; however, lack of justification for the choice of a certain outcome measure is the rule [103].

Majority of studies (63 out of $73,86.30 \%$ ) use more than one outcome measures, a procedure that has been proposed to enable comparison across trials [99,104]. However, the use of multiple outcome measurements requirerequires the a priori definition of the primary outcome, which was the case only in 14 studies out of the 64 . It is also interesting, that 34 of the RCTs use at least three outcome measures, which shows a relatively wide range of domains targeted and also increases the possibility of results ought to randomness.

\subsection{Time Course}

Relatively little is known about the course of tinnitus over time [7,105-107]. There are a lot of factors contributing to this. Tinnitus installation is often prolonged and there is a considerable proportion of patients that cannot clearly identify an exact date of tinnitus onset. Especially in the cases where tinnitus habituation has occurred, patients may not clearly recall or may underestimate both time of onset or severity of their tinnitus, when they are asked about it or when they are filling retrospectively a relevant questionnaire. A considerable proportion with total relapse might not even contact health services, and therefore never be recorded, which means that estimation of the course of tinnitus over time is in current studies at based assessed retrospectively by questionnaires, which are subject to recall bias as well as to suboptimal phrasing of the relevant questions in the self-filled questionnaires. Consequently, there is lack of reliable information about the actual incidence, the course and the profile of the patients who experience tinnitus for a short period of time and then stop experiencing it. On top of this, patient trajectories differ strongly across countries depending in the health system.

Tinnitus is usually dichotomized into acute and chronic; however, recent European guidelines have also included the sub-acute type (from 3 to 6 months), in order to reflect the transition from acute to chronic tinnitus [1]. However, all these definitions are arbitrary, and little is known about differences in the pathophysiology of acute and chronic tinnitus and the time when this transition occurs. It is remarkable that only two of the RCTs identified focused on acute tinnitus $[50,74]$.

The majority of the studies (58 of 73) clearly defines a minimum time interval from tinnitus onset, however variability in time intervals is large. Eleven trials set as minimum duration 3 months and 22 the 6 months interval, whereas a wide range of smaller or larger intervals occur. This variance may be relevant for the tinnitus course, since a recent 
systematic review has indicated a statistically significant decrease in the impact of tinnitus over time, although clinical significance could not be interpreted due to heterogeneity [108]. This practically means that in the comparison between RCTs differences in the tinnitus duration might matter.

\subsection{Trial Design and Results}

As expected, there is a large heterogeneity among the RCTs included. About one third of the included studies examine the efficacy of pharmaceutical agents either as sy-stemic or as topical administration. Second most common topic is various types of TMS, whereas 10 focus on CBT either face to face or online, 5 on non-CBT psychological interventions, 7 on HAs (alone or in combination with motivational interview), and 8 on sound therapy. Finally, acupuncture and laser beam have also been evaluated as monotherapy for chronic tinnitus $[25,27,34,43,51,53,66]$. The variance of the interventions with respect to their intended mechanisms, targets and duration should have led to different trial designs in terms of outcome measures as well as follow up schedule. For instance, TMS is usually implemented in strict and well-defined time periods (typically one to two weeks), whereas CBT is an intervention lasting several weeks and should be finished before the effect can be evaluated. At the same time, HAs have a continuous and possibly long-lasting effect. This is not reflected in the design of the studies, since criteria in regards to tinnitus onset, follow up duration and outcome measures are more or less equally distributed in these sub-groups of RCTs.

It is noteworthy that two third of control groups use different types of methods in order to be non-interventional: placebo, sham devices or interventions, participants from the waiting list or usual care. Ideally, the recommendation is to use best available treatment instead of placebo, at least for pharmacological studies. This is probably not applicable in the tinnitus field, since universally acceptable treatment is pending [109]. Hence, majority of the remaining studies use as control arms active interventions of the same discipline (different TMS protocols, HA fitting parameters, stimuli used for sound therapies). Very few RCTs compare two totally independent interventions. This design should be considered in future studies, because on top of efficacy superiority, it could potentially identify profiles of patients who could be more prompt to one intervention compared to other.

Although evaluation of results was not within the core scopes of this review, it should be mentioned, that roughly one third of the RCTs concluded that the intervention tested was considered effective. RCTs targeting CBT and different types of sound therapy re-presented more than one third of the RCTs with a positive outcome, whereas their proportion in the whole body of RCTs was significantly lower (13.6\% and $10.96 \%$, respectively). All other types of interventions had at least one clinical trial with a positive result (superiority against the control intervention).

One important aspect is that with regards to several RCTs in which different active interventions were compared, it remains unclear whether the results differ from placebo. Even if there were significant within arm comparisons for all investigated interventions, one cannot unambiguously differentiate between spontaneous improvement and an effect of the investigated intervention.

Moreover, with very few exceptions, only statistical and not clinical significance was examined, and any minimum benefit considered as significant was set ad hoc.

\section{Conclusions}

Tinnitus is a clinical enigma for many reasons, most of which are intrinsic. RCTs in tinnitus field suffer from many methodological flaws, including lack of strict and welldefined inclusion criteria, failure to eliminate confounders like hearing loss, stress, and tinnitus duration, variance in outcome measures and interventions, and barriers in design. Future RCTs should clearly set a minimum level of tinnitus related annoyance, range of 
age and tinnitus duration, hearing ability, as inclusion criteria, choose appropriate outcome measures for the intervention tested and interpret clinical on top of statistical signi-ficance.

Design of future trials should take into account issues, barriers and problems identified in this review. Patients with intermittent tinnitus should probably be included in different studies than those with tinnitus lasting all day. Although tinnitus onset might not be always clear, the majority of studies seem targeting chronic tinnitus ( $>6$ months) and a gap of studies targeting acute tinnitus has been identified. A strict range of scores in the outcome measures should be set as inclusion criteria and the results of the trials should be clearly generalized in the relevant population. Outcome measures should be selected based on intervention and targeting related tinnitus dimensions, in line with current literature [92]. Comorbidity of hearing loss and its level should be also taken into account, not only as inclusion criterion, but also as a potential profiling factor for patients and treatment response. Tinnitus pitch, minimum masking level, and residual inhibition should also be considered as prognostic factors. Patients should be monitored for at least six months, in order to ensure stabilized results. Overall, trial designs and analysis should overcome the classic schema of comparing two interventions at two points: tinnitus is complex and heterogenous and requires identification of certain subgroups who are prone to certain treatments and of prognostic factors for treatment outcome.

Author Contributions: Conceptualization, D.K. and W.S.; methodology, E.I. and D.K.; investigation, D.K., N.M., A.T., and E.V.; writing-original draft preparation, D.K., E.V., A.T., and E.I.; writingreview and editing, D.K., W.S., and B.L.; supervision, D.K. All authors have read and agreed to the published version of the manuscript.

Funding: This work is supported by the European Union's Horizon 2020 Research and Innovation Programme UNITI-Unification of Treatments and Interventions for Tinnitus Patients, Grant Agreement Number 848261.

Conflicts of Interest: The authors declare no conflict of interest.

\section{References}

1. Cima, R.F.F.; Mazurek, B.; Haider, H.; Kikidis, D.; Lapira, A.; Noreña, A.; Hoare, D.J. A Multidisciplinary European Guideline for Tinnitus: Diagnostics, Assessment, and Treatment. HNO 2019, 67, 10-42. [CrossRef] [PubMed]

2. De Ridder, D.; Schlee, W.; Vanneste, S.; Londero, A.; Weisz, N.; Kleinjung, T.; Shekhawat, G.S.; Elgoyhen, A.B.; Song, J.-J.; Andersson, G.; et al. Chapter 1-Tinnitus and tinnitus disorder: Theoretical and operational definitions (an international multidisciplinary proposal). In Progress in Brain Research; Schlee, W., Langguth, B., Kleinjung, T., Vanneste, S., De Ridder, D., Eds.; Tinnitus-An Interdisciplinary Approach Towards Individualized Treatment: From Heterogeneity to Personalized Medicine; Elsevier: Amsterdam, The Netherlands, 2021; Volume 260, pp. 1-25.

3. Cima, R.F.F. Bothersome Tinnitus. HNO 2018, 66, 369-374. [CrossRef] [PubMed]

4. Bhatt, J.M.; Lin, H.W.; Bhattacharyya, N. Tinnitus Epidemiology: Prevalence, Severity, Exposures And Treatment Patterns In The United States. JAMA Otolaryngol. Head Neck Surg. 2016, 142, 959-965. [CrossRef]

5. Hesse, G. Evidence and Evidence Gaps in Tinnitus Therapy. GMS Curr. Top. Otorhinolaryngol. Head Neck Surg. 2016, 15. [CrossRef]

6. Hoare, D.J.; Kowalkowski, V.L.; Kang, S.; Hall, D.A. Systematic Review and Meta-Analyses of Randomized Controlled Trials Examining Tinnitus Management. Laryngoscope 2011, 121, 1555-1564. [CrossRef]

7. Simoes, J.; Neff, P.; Schoisswohl, S.; Bulla, J.; Schecklmann, M.; Harrison, S.; Vesala, M.; Langguth, B.; Schlee, W. Toward Personalized Tinnitus Treatment: An Exploratory Study Based on Internet Crowdsensing. Front. Public Health 2019, 7. [CrossRef]

8. Arienti, C.; Armijo-Olivo, S.; Minozzi, S.; Lazzarini, S.G.; Patrini, M.; Negrini, S. 60 Methodological Issues in Rehabilitation Research: A Scoping Review. BMJ EBM 2019, 24, A35. [CrossRef]

9. Langguth, B.; Landgrebe, M.; Schlee, W.; Schecklmann, M.; Vielsmeier, V.; Steffens, T.; Staudinger, S.; Frick, H.; Frick, U. Different Patterns of Hearing Loss among Tinnitus Patients: A Latent Class Analysis of a Large Sample. Front. Neurol. 2017, 8. [CrossRef]

10. Elgoyhen, A.B.; Langguth, B.; De Ridder, D.; Vanneste, S. Tinnitus: Perspectives from Human Neuroimaging. Nat. Rev. Neurosci. 2015, 16, 632-642. [CrossRef]

11. Probst, T.; Pryss, R.C.; Langguth, B.; Rauschecker, J.P.; Schobel, J.; Reichert, M.; Spiliopoulou, M.; Schlee, W.; Zimmermann, J. Does Tinnitus Depend on Time-of-Day? An Ecological Momentary Assessment Study with the "TrackYourTinnitus" Application. Front. Aging Neurosci. 2017, 9. [CrossRef] [PubMed]

12. Winfried, S.; Stefan, S.; Susanne, S.; Axel, S.; Astrid, L.; Berthold, L.; Martin, S.; Jorge, S.; Patrick, N.; Steven, M.; et al. Towards a unification of treatments and interventions for tinnitus patients: The EU research and innovation action UNITI. In Progress in Brain Research; Elsevier: Amsterdam, The Netherlands, 2021. 
13. Shamseer, L.; Moher, D.; Clarke, M.; Ghersi, D.; Liberati, A.; Petticrew, M.; Shekelle, P.; Stewart, L.A.; The PRISMA-P Group. Preferred Reporting Items for Systematic Review and Meta-Analysis Protocols (PRISMA-P) 2015: Elaboration and Explanation. BMJ 2015, 349, g7647. [CrossRef]

14. Hopewell, S.; Clarke, M.; Lusher, A.; Lefebvre, C.; Westby, M. A Comparison of Handsearching versus MEDLINE Searching to Identify Reports of Randomized Controlled Trials. Stat. Med. 2002, 21, 1625-1634. [CrossRef]

15. Buscemi, N.; Hartling, L.; Vandermeer, B.; Tjosvold, L.; Klassen, T.P. Single Data Extraction Generated More Errors than Double Data Extraction in Systematic Reviews. J. Clin. Epidemiol. 2006, 59, 697-703. [CrossRef] [PubMed]

16. Anders, M.; Dvorakova, J.; Rathova, L.; Havrankova, P.; Pelcova, P.; Vaneckova, M.; Jech, R.; Holcat, M.; Seidl, Z.; Raboch, J. Efficacy of Repetitive Transcranial Magnetic Stimulation for the Treatment of Refractory Chronic Tinnitus: A Randomized, Placebo Controlled Study. Neuro Endocrinol. Lett. 2010, 31, 238-249.

17. Biesinger, E.; Kipman, U.; Schätz, S.; Langguth, B. Qigong for the Treatment of Tinnitus: A Prospectiverandomized Controlled Study. J. Psychosom. Res. 2010, 69, 299-304. [CrossRef] [PubMed]

18. Dehkordi, M.A.; Abolbashari, S.; Taheri, R.; Einolghozati, S. Efficacy of Gabapentin on Subjective Idiopathic Tinnitus: A Randomized, Double-Blind, Placebo-Controlled Trial. Ear Nose Throat J. 2011, 90, 150-158. [CrossRef]

19. Sziklai, I.; Szilvássy, J.; Szilvássy, Z. Tinnitus Control by Dopamine Agonist Pramipexole in Presbycusis Patients: A Randomized, Placebo-Controlled, Double-Blind Study. Laryngoscope 2011, 121, 888-893. [CrossRef] [PubMed]

20. Westin, V.Z.; Schulin, M.; Hesser, H.; Karlsson, M.; Noe, R.Z.; Olofsson, U.; Stalby, M.; Wisung, G.; Andersson, G. Acceptance and Commitment Therapy versus Tinnitus Retraining Therapy in the Treatment of Tinnitus: A Randomised Controlled Trial. Behav. Res. Ther. 2011, 49, 737-747. [CrossRef]

21. Cima, R.F.F.; Maes, I.H.; Joore, M.A.; Scheyen, D.J.W.M.; El Refaie, A.; Baguley, D.M.; Anteunis, L.J.C.; van Breukelen, G.J.P.; Vlaeyen, J.W.S. Specialised Treatment Based on Cognitive Behaviour Therapy versus Usual Care for Tinnitus: A Randomised Controlled Trial. Lancet 2012, 379, 1951-1959. [CrossRef]

22. Han, S.-S.; Nam, E.-C.; Won, J.Y.; Lee, K.U.; Chun, W.; Choi, H.K.; Levine, R.A. Clonazepam Quiets Tinnitus: A Randomised Crossover Study with Ginkgo Biloba. J. Neurol. Neurosurg. Psychiatry 2012, 83, 821-827. [CrossRef]

23. Hesser, H.; Gustafsson, T.; Lundén, C.; Henrikson, O.; Fattahi, K.; Johnsson, E.; Zetterqvist Westin, V.; Carlbring, P.; Mäki-Torkko, E.; Kaldo, V.; et al. A Randomized Controlled Trial of Internet-Delivered Cognitive Behavior Therapy and Acceptance and Commitment Therapy in the Treatment of Tinnitus. J. Consult. Clin. Psychol. 2012, 80, 649-661. [CrossRef]

24. Hoare, D.J.; Kowalkowski, V.L.; Hall, D.A. Effects of Frequency Discrimination Training on Tinnitus: Results from Two Randomised Controlled Trials. J. Assoc. Res. Otolaryngol. 2012, 13, 543-559. [CrossRef] [PubMed]

25. Jeon, S.W.; Kim, K.S.; Nam, H.J. Long-Term Effect of Acupuncture for Treatment of Tinnitus: A Randomized, Patient- and Assessor-Blind, Sham-Acupuncture-Controlled, Pilot Trial. J. Altern. Complement. Med. 2012, 18, 693-699. [CrossRef] [PubMed]

26. Kreuzer, P.M.; Goetz, M.; Holl, M.; Schecklmann, M.; Landgrebe, M.; Staudinger, S.; Langguth, B. Mindfulness-and BodyPsychotherapy-Based Group Treatment of Chronic Tinnitus: A Randomized Controlled Pilot Study. BMC Complement. Altern. Med. 2012, 12, 235. [CrossRef] [PubMed]

27. Ngao, C.F.; Tan, T.S.; Narayanan, P.; Raman, R. The Effectiveness of Transmeatal Low-Power Laser Stimulation in Treating Tinnitus. Eur. Arch. Otorhinolaryngol. 2014, 271, 975-980. [CrossRef] [PubMed]

28. Plewnia, C.; Vonthein, R.; Wasserka, B.; Arfeller, C.; Naumann, A.; Schraven, S.P.; Plontke, S.K. Treatment of Chronic Tinnitus with $\theta$ Burst Stimulation: A Randomized Controlled Trial. Neurology 2012, 78, 1628-1634. [CrossRef]

29. Rocha, C.B.; Sanchez, T.G. Efficacy of Myofascial Trigger Point Deactivation for Tinnitus Control. Braz. J. Otorhinolaryngol. 2012, 78, 21-26. [CrossRef]

30. Tass, P.A.; Adamchic, I.; Freund, H.-J.; von Stackelberg, T.; Hauptmann, C. Counteracting Tinnitus by Acoustic Coordinated Reset Neuromodulation. Restor. Neurol. Neurosci. 2012, 30, 137-159. [CrossRef]

31. Choi, S.J.; Lee, J.B.; Lim, H.J.; In, S.M.; Kim, J.-Y.; Bae, K.H.; Choung, Y.-H. Intratympanic Dexamethasone Injection for Refractory Tinnitus: Prospective Placebo-Controlled Study. Laryngoscope 2013, 123, 2817-2822. [CrossRef]

32. Coelho, C.; Witt, S.A.; Ji, H.; Hansen, M.R.; Gantz, B.; Tyler, R. Zinc to Treat Tinnitus in the Elderly: A Randomized Placebo Controlled Crossover Trial. Otol. Neurotol. 2013, 34, 1146-1154. [CrossRef]

33. Hoekstra, C.E.L.; Versnel, H.; Neggers, S.F.W.; Niesten, M.E.F.; van Zanten, G.A. Bilateral Low-Frequency Repetitive Transcranial Magnetic Stimulation of the Auditory Cortex in Tinnitus Patients Is Not Effective: A Randomised Controlled Trial. Audiol. Neurootol. 2013, 18, 362-373. [CrossRef] [PubMed]

34. Mollasadeghi, A.; Mirmohammadi, S.J.; Mehrparvar, A.H.; Davari, M.H.; Shokouh, P.; Mostaghaci, M.; Baradaranfar, M.H.; Bahaloo, M. Efficacy of Low-Level Laser Therapy in the Management of Tinnitus Due to Noise-Induced Hearing Loss: A DoubleBlind Randomized Clinical Trial. Sci. World J. 2013, 2013. [CrossRef] [PubMed]

35. Nyenhuis, N.; Zastrutzki, S.; Weise, C.; Jäger, B.; Kröner-Herwig, B. The Efficacy of Minimal Contact Interventions for Acute Tinnitus: A Randomised Controlled Study. Cogn. Behav. Ther. 2013, 42, 127-138. [CrossRef] [PubMed]

36. Sönmez, O.; Külahlı, I.; Vural, A.; Sahin, M.I.; Aydın, M. The Evaluation of Ozone and Betahistine in the Treatment of Tinnitus. Eur. Arch. Otorhinolaryngol. 2013, 270, 1999-2006. [CrossRef] [PubMed]

37. Taslimi, S.; Vahidi, H.; Pourvaziri, A.; Modabbernia, A.; Fallah, A.Y.; Yazdani, N.; Taslimi, N.; Hosseini, M.; Zarandi, M.M. Ondansetron in Patients with Tinnitus: Randomized Double-Blind Placebo-Controlled Study. Eur. Arch. Otorhinolaryngol. 2013, 270, 1635-1641. [CrossRef] [PubMed] 
38. Dos Santos, G.M.; Bento, R.F.; de Medeiros, I.R.T.; Oiticcica, J.; da Silva, E.C.; Penteado, S. The Influence of Sound Generator Associated With Conventional Amplification for Tinnitus Control: Randomized Blind Clinical Trial. Trends Hear. 2014, 18, 233121651454265. [CrossRef]

39. Hoare, D.J.; Van Labeke, N.; McCormack, A.; Sereda, M.; Smith, S.; Al Taher, H.; Kowalkowski, V.L.; Sharples, M.; Hall, D.A. Gameplay as a Source of Intrinsic Motivation in a Randomized Controlled Trial of Auditory Training for Tinnitus. PLoS ONE 2014, 9, e107430. [CrossRef]

40. Jasper, K.; Weise, C.; Conrad, I.; Andersson, G.; Hiller, W.; Kleinstäuber, M. Internet-Based Guided Self-Help versus Group Cognitive Behavioral Therapy for Chronic Tinnitus: A Randomized Controlled Trial. Psychother. Psychosom. 2014, 83, 234-246. [CrossRef] [PubMed]

41. Shekhawat, G.S.; Searchfield, G.D.; Stinear, C.M. Randomized Trial of Transcranial Direct Current Stimulation and Hearing Aids for Tinnitus Management. Neurorehabilit. Neural Repair 2014, 28, 410-419. [CrossRef]

42. Teismann, H.; Wollbrink, A.; Okamoto, H.; Schlaug, G.; Rudack, C.; Pantev, C. Combining Transcranial Direct Current Stimulation and Tailor-Made Notched Music Training to Decrease Tinnitus-Related Distress-A Pilot Study. PLoS ONE 2014, 9, e89904. [CrossRef]

43. Dehkordi, M.A.; Einolghozati, S.; Ghasemi, S.M.; Abolbashari, S.; Meshkat, M.; Behzad, H. Effect of Low-Level Laser Therapy in the Treatment of Cochlear Tinnitus: A Double-Blind, Placebo-Controlled Study. Ear Nose Throat J. 2015, 94, 32-36. [PubMed]

44. Bilici, S.; Yigit, O.; Taskin, U.; Gor, A.P.; Yilmaz, E.D. Medium-Term Results of Combined Treatment with Transcranial Magnetic Stimulation and Antidepressant Drug for Chronic Tinnitus. Eur. Arch. Otorhinolaryngol. 2015, 272, 337-343. [CrossRef] [PubMed]

45. Folmer, R.L.; Theodoroff, S.M.; Casiana, L.; Shi, Y.; Griest, S.; Vachhani, J. Repetitive Transcranial Magnetic Stimulation Treatment for Chronic Tinnitus: A Randomized Clinical Trial. JAMA Otolaryngol. Head Neck Surg. 2015, 141, 716-722. [CrossRef]

46. Kreuzer, P.M.; Lehner, A.; Schlee, W.; Vielsmeier, V.; Schecklmann, M.; Poeppl, T.B.; Landgrebe, M.; Rupprecht, R.; Langguth, B. Combined RTMS Treatment Targeting the Anterior Cingulate and the Temporal Cortex for the Treatment of Chronic Tinnitus. Sci. Rep. 2015, 5, 18028. [CrossRef]

47. Malinvaud, D.; Londero, A.; Niarra, R.; Peignard, P.; Warusfel, O.; Viaud-Delmon, I.; Chatellier, G.; Bonfils, P. Auditory and Visual 3D Virtual Reality Therapy as a New Treatment for Chronic Subjective Tinnitus: Results of a Randomized Controlled Trial. Hear. Res. 2016, 333, 127-135. [CrossRef]

48. Pal, N.; Maire, R.; Stephan, M.A.; Herrmann, F.R.; Benninger, D.H. Transcranial Direct Current Stimulation for the Treatment of Chronic Tinnitus: A Randomized Controlled Study. Brain Stimul. Basic Transl. Clin. Res. Neuromodulation 2015, 8, 1101-1107. [CrossRef]

49. Thabit, M.N.; Fouad, N.; Shahat, B.; Youssif, M. Combined Central and Peripheral Stimulation for Treatment of Chronic Tinnitus: A Randomized Pilot Study. Neurorehabilit. Neural Repair 2015, 29, 224-233. [CrossRef] [PubMed]

50. Albu, S.; Nagy, A.; Doros, C.; Marceanu, L.; Cozma, S.; Musat, G.; Trabalzini, F. Treatment of Meniere's Disease with Intratympanic Dexamethazone plus High Dosage of Betahistine. Am. J. Otolaryngol. 2016, 37, 225-230. [CrossRef] [PubMed]

51. Doi, M.Y.; Tano, S.S.; Schultz, A.R.; Borges, R.; de Moraes Marchiori, L.L. Effectiveness of Acupuncture Therapy as Treatment for Tinnitus: A Randomized Controlled Trial. Braz. J. Otorhinolaryngol. 2016, 82, 458-465. [CrossRef] [PubMed]

52. Henry, J.A.; Stewart, B.J.; Griest, S.; Kaelin, C.; Zaugg, T.L.; Carlson, K. Multisite Randomized Controlled Trial to Compare Two Methods of Tinnitus Intervention to Two Control Conditions. Ear Hear. 2016, 37, e346-e359. [CrossRef]

53. Laureano, M.R.; Onishi, E.T.; Bressan, R.A.; Neto, P.B.; Castiglioni, M.L.V.; Batista, I.R.; Reis, M.A.; Garcia, M.V.; de Andrade, A.N.; Sanchez, M.L.; et al. The Effectiveness of Acupuncture as a Treatment for Tinnitus: A Randomized Controlled Trial Using (99m)Tc-ECD SPECT. Eur. Radiol. 2016, 26, 3234-3242. [CrossRef] [PubMed]

54. Lehner, A.; Schecklmann, M.; Greenlee, M.W.; Rupprecht, R.; Langguth, B. Triple-Site RTMS for the Treatment of Chronic Tinnitus: A Randomized Controlled Trial. Sci. Rep. 2016, 6, 22302. [CrossRef]

55. Li, S.-A.; Bao, L.; Chrostowski, M. Investigating the Effects of a Personalized, Spectrally Altered Music-Based Sound Therapy on Treating Tinnitus: A Blinded, Randomized Controlled Trial. Audiol. Neurootol. 2016, 21, 296-304. [CrossRef]

56. Lim, H.W.; Kim, T.S.; Kang, W.S.; Song, C.I.; Baek, S.; Chung, J.W. Effect of a 4-Week Treatment with Cilostazol in Patients with Chronic Tinnitus: A Randomized, Prospective, Placebo-Controlled, Double-Blind, Pilot Study. J. Int. Adv. Otol. 2016, 12, 170-176. [CrossRef] [PubMed]

57. Rojas-Roncancio, E.; Tyler, R.; Jun, H.-J.; Wang, T.-C.; Ji, H.; Coelho, C.; Witt, S.; Hansen, M.R.; Gantz, B.J. Manganese and Lipoflavonoid Plus®to Treat Tinnitus: A Randomized Controlled Trial. J. Am. Acad. Audiol. 2016, 27, 661-668. [CrossRef] [PubMed]

58. Roland, L.T.; Peelle, J.E.; Kallogjeri, D.; Nicklaus, J.; Piccirillo, J.F. The Effect of Noninvasive Brain Stimulation on Neural Connectivity in Tinnitus: A Randomized Trial. Laryngoscope 2016, 126, 1201-1206. [CrossRef]

59. Singh, C.; Kawatra, R.; Gupta, J.; Awasthi, V.; Dungana, H. Therapeutic Role of Vitamin B12 in Patients of Chronic Tinnitus: A Pilot Study. Noise Health 2016, 18, 93-97. [CrossRef]

60. Stein, A.; Wunderlich, R.; Lau, P.; Engell, A.; Wollbrink, A.; Shaykevich, A.; Kuhn, J.-T.; Holling, H.; Rudack, C.; Pantev, C. Clinical Trial on Tonal Tinnitus with Tailor-Made Notched Music Training. BMC Neurol. 2016, 16. [CrossRef]

61. Weise, C.; Kleinstäuber, M.; Andersson, G. Internet-Delivered Cognitive-Behavior Therapy for Tinnitus: A Randomized Controlled Trial. Psychosom. Med. 2016, 78, 501-510. [CrossRef] 
62. Wise, K.; Kobayashi, K.; Magnusson, J.; Welch, D.; Searchfield, G.D. Randomized Controlled Trial of a Perceptual Training Game for Tinnitus Therapy. Games Health J. 2016, 5, 141-149. [CrossRef]

63. Zarenoe, R.; Söderlund, L.L.; Andersson, G.; Ledin, T. Motivational Interviewing as an Adjunct to Hearing Rehabilitation for Patients with Tinnitus: A Randomized Controlled Pilot Trial. J. Am. Acad. Audiol. 2016, 27, 669-676. [CrossRef] [PubMed]

64. Elzayat, S.; El-Sherif, H.; Hegazy, H.; Gabr, T.; El-Tahan, A.-R. Tinnitus: Evaluation of Intratympanic Injection of Combined Lidocaine and Corticosteroids. ORL J. Otorhinolaryngol. Relat. Spec. 2016, 78, 159-166. [CrossRef]

65. Kallogjeri, D.; Piccirillo, J.F.; Spitznagel, E.; Hale, S.; Nicklaus, J.E.; Hardin, F.M.; Shimony, J.S.; Coalson, R.S.; Schlaggar, B.L. Cognitive Training for Adults With Bothersome Tinnitus. JAMA Otolaryngol. Head Neck Surg. 2017, 143, 443-451. [CrossRef] [PubMed]

66. Kim, B.H.; Kim, K.; Nam, H.J. A Comparative Study on the Effects of Systemic Manual Acupuncture, Periauricular Electroacupuncture, and Digital Electroacupuncture to Treat Tinnitus: A Randomized, Paralleled, Open-Labeled Exploratory Trial. BMC Complement. Altern. Med. 2017, 17. [CrossRef]

67. Landgrebe, M.; Hajak, G.; Wolf, S.; Padberg, F.; Klupp, P.; Fallgatter, A.J.; Polak, T.; Höppner, J.; Haker, R.; Cordes, J.; et al. 1-Hz RTMS in the Treatment of Tinnitus: A Sham-Controlled, Randomized Multicenter Trial. Brain Stimul. 2017, 10, 1112-1120. [CrossRef] [PubMed]

68. McKenna, L.; Marks, E.M.; Hallsworth, C.A.; Schaette, R. Mindfulness-Based Cognitive Therapy as a Treatment for Chronic Tinnitus: A Randomized Controlled Trial. Psychother. Psychosom. 2017, 86, 351-361. [CrossRef]

69. Arif, M.; Sadlier, M.; Rajenderkumar, D.; James, J.; Tahir, T. A Randomised Controlled Study of Mindfulness Meditation versus Relaxation Therapy in the Management of Tinnitus. J. Laryngol. Otol. 2017, 131, 501-507. [CrossRef] [PubMed]

70. Beukes, E.W.; Baguley, D.M.; Allen, P.M.; Manchaiah, V.; Andersson, G. Guided Internet-Based versus Face-to-Face Clinical Care in the Management of Tinnitus: Study Protocol for a Multi-Centre Randomised Controlled Trial. Trials 2017, 18. [CrossRef]

71. Sahlsten, H.; Virtanen, J.; Joutsa, J.; Niinivirta-Joutsa, K.; Löyttyniemi, E.; Johansson, R.; Paavola, J.; Taiminen, T.; Sjösten, N.; Salonen, J.; et al. Electric Field-Navigated Transcranial Magnetic Stimulation for Chronic Tinnitus: A Randomized, PlaceboControlled Study. Int. J. Audiol. 2017, 56, 692-700. [CrossRef] [PubMed]

72. Theodoroff, S.M.; Griest, S.E.; Folmer, R.L. Transcranial Magnetic Stimulation for Tinnitus: Using the Tinnitus Functional Index to Predict Benefit in a Randomized Controlled Trial. Trials 2017, 18. [CrossRef]

73. Tyler, R.; Cacace, A.; Stocking, C.; Tarver, B.; Engineer, N.; Martin, J.; Deshpande, A.; Stecker, N.; Pereira, M.; Kilgard, M.; et al. Vagus Nerve Stimulation Paired with Tones for the Treatment of Tinnitus: A Prospective Randomized Double-Blind Controlled Pilot Study in Humans. Sci. Rep. 2017, 7. [CrossRef]

74. Lee, H.-J.; Kim, M.-B.; Yoo, S.-Y.; Park, S.N.; Nam, E.-C.; Moon, I.S.; Lee, H.-K. Clinical Effect of Intratympanic Dexamethasone Injection in Acute Unilateral Tinnitus: A Prospective, Placebo-Controlled, Multicenter Study. Laryngoscope 2018, 128, 184-188. [CrossRef]

75. Beukes, E.W.; Andersson, G.; Allen, P.M.; Manchaiah, V.; Baguley, D.M. Effectiveness of Guided Internet-Based Cognitive Behavioral Therapy vs Face-to-Face Clinical Care for Treatment of Tinnitus. JAMA Otolaryngol. Head Neck Surg. 2018, 144, 1126-1133. [CrossRef]

76. Abtahi, H.; Okhovvat, A.; Heidari, S.; Gharagazarloo, A.; Mirdamadi, M.; Nilforoush, M.H.; Ghazavi, H. Effect of Transcranial Direct Current Stimulation on Short-Term and Long-Term Treatment of Chronic Tinnitus. Am. J. Otolaryngol. 2018, 39, 94-96. [CrossRef]

77. El Beaino, M.; McCaskey, M.K.; Eter, E. Sulodexide Monotherapy in Chronic Idiopathic Subjective Tinnitus: A Randomized Controlled Trial. Otolaryngol. Head Neck Surg. 2018, 158, 1107-1112. [CrossRef] [PubMed]

78. Hong, H.Y.; Karadaghy, O.; Kallogjeri, D.; Brown, F.T.; Yee, B.; Piccirillo, J.F.; Nagele, P. Effect of Nitrous Oxide as a Treatment for Subjective, Idiopathic, Nonpulsatile Bothersome Tinnitus: A Randomized Clinical Trial. JAMA Otolaryngol. Head Neck Surg. 2018, 144, 781-787. [CrossRef] [PubMed]

79. Godbehere, J.; Sandhu, J.; Evans, A.; Twigg, V.; Scivill, I.; Ray, J.; Barker, A. Treatment of Tinnitus Using Theta Burst Based Repetitive Transcranial Magnetic Stimulation-A Single Blinded Randomized Control Trial. Otol. Neurotol. 2019, 40, S38. [CrossRef]

80. Hall, D.A.; Ray, J.; Watson, J.; Sharman, A.; Hutchison, J.; Harris, P.; Daniel, M.; Millar, B.; Large, C.H. A Balanced Randomised Placebo Controlled Blinded Phase IIa Multi-Centre Study to Investigate the Efficacy and Safety of AUT00063 versus Placebo in Subjective Tinnitus: The QUIET-1 Trial. Hear. Res. 2019, 377, 153-166. [CrossRef]

81. Li, J.; Jin, J.; Xi, S.; Zhu, Q.; Chen, Y.; Huang, M.; He, C. Clinical Efficacy of Cognitive Behavioral Therapy for Chronic Subjective Tinnitus. Am. J. Otolaryngol. 2019, 40, 253-256. [CrossRef] [PubMed]

82. Noh, T.-S.; Kyong, J.-S.; Park, M.K.; Lee, J.H.; Oh, S.H.; Chung, C.K.; Kim, J.S.; Suh, M.-W. Treatment Outcome of Auditory and Frontal Dual-Site RTMS in Tinnitus Patients and Changes in Magnetoencephalographic Functional Connectivity after RTMS: Double-Blind Randomized Controlled Trial. Audiol. Neurootol. 2019, 24, 293-298. [CrossRef] [PubMed]

83. Procházková, K.; Šejna, I.; Skutil, J.; Hahn, A. Ginkgo Biloba Extract EGb 761®versus Pentoxifylline in Chronic Tinnitus: A Randomized, Double-Blind Clinical Trial. Int. J. Clin. Pharm. 2018, 40, 1335-1341. [CrossRef]

84. Radunz, C.L.; Okuyama, C.E.; Branco-Barreiro, F.C.A.; Pereira, R.M.S.; Diniz, S.N. Clinical Randomized Trial Study of Hearing Aids Effectiveness in Association with Ginkgo Biloba Extract (EGb 761) on Tinnitus Improvement. Braz. J. Otorhinolaryngol. 2019. [CrossRef] 
85. Sahlsten, H.; Holm, A.; Rauhala, E.; Takala, M.; Löyttyniemi, E.; Karukivi, M.; Nikkilä, J.; Ylitalo, K.; Paavola, J.; Johansson, R.; et al. Neuronavigated Versus Non-Navigated Repetitive Transcranial Magnetic Stimulation for Chronic Tinnitus: A Randomized Study. Trends Hear. 2019, 23, 2331216518822198. [CrossRef] [PubMed]

86. Scherer, R.W.; Formby, C. Effect of Tinnitus Retraining Therapy vs Standard of Care on Tinnitus-Related Quality of Life. JAMA Otolaryngol. Head Neck Surg. 2019. [CrossRef]

87. Yakunina, N.; Lee, W.H.; Ryu, Y.-J.; Nam, E.-C. Tinnitus Suppression Effect of Hearing Aids in Patients With High-Frequency Hearing Loss: A Randomized Double-Blind Controlled Trial. Otol. Neurotol. 2019, 40, 865-871. [CrossRef] [PubMed]

88. Tutar, B.; Atar, S.; Berkiten, G.; Üstün, O.; Kumral, T.L.; Uyar, Y. The Effect of Transcutaneous Electrical Nerve Stimulation (TENS) on Chronic Subjective Tinnitus. Am. J. Otolaryngol. 2020, 41, 102326. [CrossRef]

89. Chessum, L.; Matern, M.S.; Kelly, M.C.; Johnson, S.L.; Ogawa, Y.; Milon, B.; McMurray, M.; Driver, E.C.; Parker, A.; Song, Y.; et al. Helios Is a Key Transcriptional Regulator of Outer Hair Cell Maturation. Nature 2018, 563, 696-700. [CrossRef] [PubMed]

90. Fettiplace, R. Hair Cell Transduction, Tuning, and Synaptic Transmission in the Mammalian Cochlea. In Comprehensive Physiology; Terjung, R., Ed.; John Wiley \& Sons, Inc.: Hoboken, NJ, USA, 2017; pp. 1197-1227. ISBN 978-0-470-65071-4.

91. Kreuzer, P.M.; Vielsmeier, V.; Poeppl, T.B.; Langguth, B. A Case Report on Red Ear Syndrome with Tinnitus Successfully Treated with Transcranial Random Noise Stimulation. Pain Physician 2017, 20, E199-E205. [CrossRef]

92. Rauschecker, J.P.; May, E.S.; Maudoux, A.; Ploner, M. Frontostriatal Gating of Tinnitus and Chronic Pain. Trends Cogn. Sci. 2015, 19, 567-578. [CrossRef]

93. Coelho, C.; Figueiredo, R.; Frank, E.; Burger, J.; Schecklmann, M.; Landgrebe, M.; Langguth, B.; Elgoyhen, A.B. Reduction of Tinnitus Severity by the Centrally Acting Muscle Relaxant Cyclobenzaprine: An Open-Label Pilot Study. Audiol. Neurootol. 2012, 17, 179-188. [CrossRef]

94. Henry, J.A.; McMillan, G.; Dann, S.; Bennett, K.; Griest, S.; Theodoroff, S.; Silverman, S.P.; Whichard, S.; Saunders, G. Tinnitus Management: Randomized Controlled Trial Comparing Extended-Wear Hearing Aids, Conventional Hearing Aids, and Combination Instruments. J. Am. Acad. Audiol. 2017, 28, 546-561. [CrossRef] [PubMed]

95. Hobson, J.; Chisholm, E.; El Refaie, A. Sound therapy (masking) in the management of tinnitus in adults. In Cochrane Database of Systematic Reviews; The Cochrane Collaboration, Ed.; John Wiley \& Sons, Ltd.: Chichester, UK, 2010.

96. Mahboubi, H.; Haidar, Y.M.; Kiumehr, S.; Ziai, K.; Djalilian, H.R. Customized Versus Noncustomized Sound Therapy for Treatment of Tinnitus: A Randomized Crossover Clinical Trial. Ann. Otol. Rhinol. Laryngol. 2017, 126, 681-687. [CrossRef]

97. Schaette, R.; König, O.; Hornig, D.; Gross, M.; Kempter, R. Acoustic Stimulation Treatments against Tinnitus Could Be Most Effective When Tinnitus Pitch Is within the Stimulated Frequency Range. Hear. Res. 2010, 269, 95-101. [CrossRef]

98. Folmer, R.L.; Carroll, J.R. Long-Term Effectiveness of Ear-Level Devices for Tinnitus. Otolaryngol. Head Neck Surg. 2006, 134, 132-137. [CrossRef]

99. Langguth, B.; Goodey, R.; Azevedo, A.; Bjorne, A.; Cacace, A.; Crocetti, A.; Del Bo, L.; De Ridder, D.; Diges, I.; Elbert, T.; et al. Consensus for Tinnitus Patient Assessment and Treatment Outcome Measurement: Tinnitus Research Initiative Meeting, Regensburg, July 2006. Prog. Brain Res. 2007, 166, 525-536. [CrossRef] [PubMed]

100. Koops, E.A.; Husain, F.T;; van Dijk, P. Profiling Intermittent Tinnitus: A Retrospective Review. Int. J. Audiol. 2019, 58, 434-440. [CrossRef]

101. Axelsson, A.; Ringdahl, A. Tinnitus-A Study of Its Prevalence and Characteristics. Br. J. Audiol. 1989, 23, 53-62. [CrossRef]

102. Degeest, S.; Corthals, P.; Dhooge, I.; Keppler, H. The Impact of Tinnitus Characteristics and Associated Variables on TinnitusRelated Handicap. J. Laryngol. Otol. 2016, 130, 25-31. [CrossRef] [PubMed]

103. Hall, D.A.; Hibbert, A.; Smith, H.; Haider, H.F.; Londero, A.; Mazurek, B.; Fackrell, K. One Size Does Not Fit All: Developing Common Standards for Outcomes in Early-Phase Clinical Trials of Sound-, Psychology-, and Pharmacology-Based Interventions for Chronic Subjective Tinnitus in Adults. Trends Hear. 2019, 23. [CrossRef]

104. Landgrebe, M.; Azevedo, A.; Baguley, D.; Bauer, C.; Cacace, A.; Coelho, C.; Dornhoffer, J.; Figueiredo, R.; Flor, H.; Hajak, G.; et al. Methodological Aspects of Clinical Trials in Tinnitus: A Proposal for an International Standard. J. Psychosom. Res. 2012, 73, 112-121. [CrossRef]

105. Lan, L.; Li, J.; Chen, Y.; Chen, W.; Li, W.; Zhao, F.; Chen, G.; Liu, J.; Chen, Y.; Li, Y.; et al. Alterations of Brain Activity and Functional Connectivity in Transition from Acute to Chronic Tinnitus. Hum. Brain Mapp. 2020. [CrossRef] [PubMed]

106. Schlee, W.; Hølleland, S.; Bulla, J.; Simoes, J.; Neff, P.; Schoisswohl, S.; Woelflick, S.; Schecklmann, M.; Schiller, A.; Staudinger, S.; et al. The Effect of Environmental Stressors on Tinnitus: A Prospective Longitudinal Study on the Impact of the COVID-19 Pandemic. J. Clin. Med. 2020, 9, 2756. [CrossRef] [PubMed]

107. Vielsmeier, V.; Santiago Stiel, R.; Kwok, P.; Langguth, B.; Schecklmann, M. From Acute to Chronic Tinnitus: Pilot Data on Predictors and Progression. Front. Neurol. 2020, 11. [CrossRef]

108. Phillips, J.S.; McFerran, D.J.; Hall, D.A.; Hoare, D.J. The Natural History of Subjective Tinnitus in Adults: A Systematic Review and Meta-Analysis of No-Intervention Periods in Controlled Trials. Laryngoscope 2018, 128, 217-227. [CrossRef]

109. Castro, M. Placebo versus Best-Available-Therapy Control Group in Clinical Trials for Pharmacologic Therapies. Proc. Am. Thorac. Soc. 2007, 4, 570-573. [CrossRef] [PubMed] 